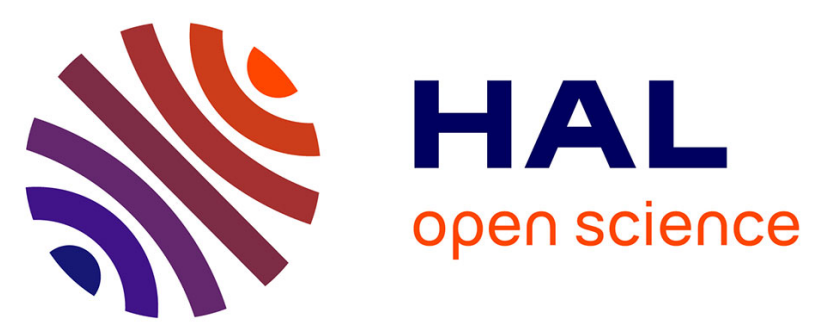

\title{
Multimodal distributions of agricultural-like sprays: A statistical analysis of drop population from a pressure-atomized spray
}

Romain Vallon, Malek Abid, Fabien Anselmet

\section{- To cite this version:}

Romain Vallon, Malek Abid, Fabien Anselmet. Multimodal distributions of agricultural-like sprays: A statistical analysis of drop population from a pressure-atomized spray. Physical Review Fluids, 2021, 6 (2), pp.023604. 10.1103/PhysRevFluids.6.023604 . hal-03141337

\section{HAL Id: hal-03141337 \\ https://hal.science/hal-03141337}

Submitted on 15 Feb 2021

HAL is a multi-disciplinary open access archive for the deposit and dissemination of scientific research documents, whether they are published or not. The documents may come from teaching and research institutions in France or abroad, or from public or private research centers.
L'archive ouverte pluridisciplinaire HAL, est destinée au dépôt et à la diffusion de documents scientifiques de niveau recherche, publiés ou non, émanant des établissements d'enseignement et de recherche français ou étrangers, des laboratoires publics ou privés. 


\title{
Multimodal distributions of agricultural-like sprays: A statistical analysis of drop population from a pressure-atomized spray
}

\author{
Romain Vallon $\odot,{ }^{*}$ Malek Abid $\odot,{ }^{\dagger}$ and Fabien Anselmet ${ }^{\ddagger}$ \\ Aix Marseille University, CNRS, Centrale Marseille, IRPHE, Marseille, France
}

(Received 9 April 2020; accepted 13 January 2021; published 11 February 2021)

\begin{abstract}
This paper focuses on the statistical analysis of a droplet population produced by a pressure-atomized jet spray, laying in the second-wind-induced regime, far behind the nozzle. The droplet size and axial velocity derived from droplet tracking velocimetry measurements are shown to follow bimodal distributions and their modeling is tackled in the framework of turbulence and of combustion applications, respectively. In addition, the existence of subsets of droplets showing specific behaviors is brought to light from the analysis of the experimental droplet-size-velocity joint probability distribution function (PDF). Such subsets can be precisely defined using the properties of the size and axialvelocity distributions. Finally, the trend of the joint PDF is depicted due to a quadratic relationship which is derived in the context of combustion and shown to work here as well, far behind the nozzle.
\end{abstract}

DOI: 10.1103/PhysRevFluids.6.023604

\section{INTRODUCTION}

Liquid jet atomization is at the heart of numerous natural and industrial systems such as ocean sprays, medication administration, and farming irrigation by aspersion. Widely used across Europe, the latter application would benefit performance gain by better understanding the atomization phenomenon. This application relies on the injection in quiescent air of a round water jet at a velocity $u_{i n j}$ through a nozzle of diameter $d_{n}$ and falls in the scope of both multiphase flows and polydisperse sprays. The phases are denoted by the subscript $k$, which takes the value $l$ for the liquid phase and $g$ for the gas phase. Classically, atomization flows are controlled by the Reynolds, Weber, and Ohnesorge numbers

$$
\operatorname{Re}_{k}=\frac{\rho_{k} u_{i n j} d_{n}}{\mu_{k}}, \quad \mathrm{We}_{k}=\frac{\rho_{k} u_{i n j}^{2} d_{n}}{\sigma_{l-g}}, \quad \mathrm{Oh}=\frac{\mu_{l}}{\sqrt{\rho_{l} d_{n} \sigma_{l-g}}},
$$

where $\rho_{k}$ and $\mu_{k}$ represent the density and the dynamic viscosity of the phase $k$, respectively, while $\sigma_{l-g}$ is the surface tension between the two phases.

Previous experiments showed the existence of five different regimes for nonassisted cylindrical liquid jets [1]. Among those, the second-wind-induced regime is not too far from the industrial application of aspersion irrigation and offers a more controlled environment for laboratory research. This high gas Weber regime is characterized by a large nozzle diameter $d_{n}>1 \mathrm{~mm}$ and sharp limits on the gas Weber number $13<\mathrm{We}_{g}<40.3$. Physically, jets belonging to this regime show a characteristic primary atomization for which small droplets are peeled off the interface near the nozzle exit. Studies have been carried out to extensively characterize this primary breakup along

\footnotetext{
*romain.vallon@univ-amu.fr

†malek.abid@univ-amu.fr

$\doteqdot$ fabien.anselmet@univ-amu.fr
} 


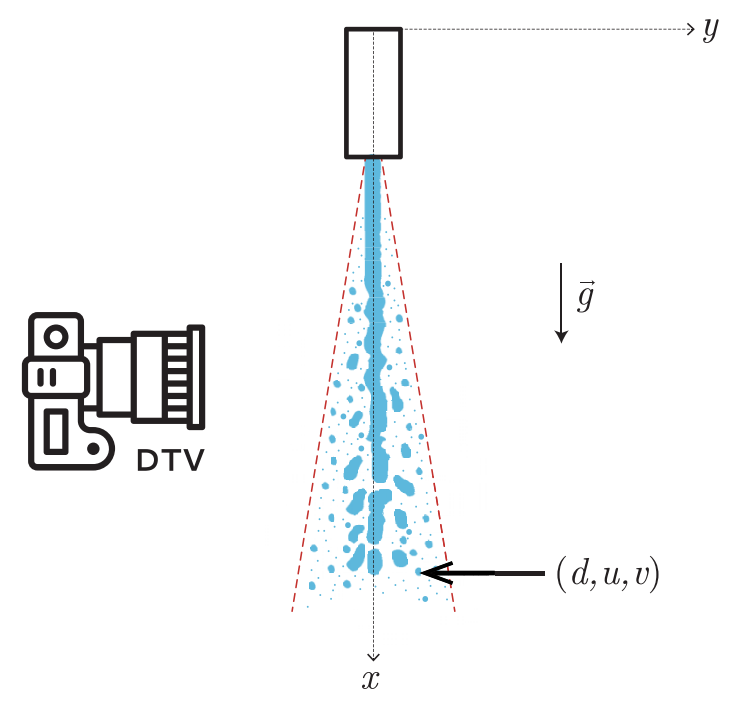

FIG. 1. Schematic of the experimental setup used by Felis et al. [8], with $\vec{g}$ the gravity field, $d$ the droplet diameter, and $(u, v)$ the droplet axial and transversal velocities, respectively, along $x$ and $y$.

the jets, before the breakup of the liquid core, including work of great importance by Faeth and co-workers [2-6], who particularly emphasized the role of turbulence by deriving a correlation for the Sauter mean diameter $d_{3,2}$ as a function of the distance to the nozzle $x$, its diameter $d_{n}$, the liquid Weber number $\mathrm{We}_{l}$, and the integral length scale of turbulence $L$. Several subregimes with different characteristic behaviors have been precisely depicted using the jet density ratio $\rho_{l} / \rho_{g}$, the liquid Weber number $\mathrm{We}_{l}$, the ratio between the Rayleigh breakup time and the aerodynamic secondary breakup time, and the degree of development of turbulence, quantified by the ratio of the nozzle length $L_{n}$ and the nozzle diameter $d_{n}$.

Following along the lines of Faeth and co-workers, later works focused on the region away from the nozzle, between the breakup of the jet liquid core and the jet dispersion zone, to study the polydisperse droplet population produced by atomization. A specific droplet tracking velocimetry (DTV) technique was used by Stevenin et al. [7] to obtain original joint size-velocity measurements which include at the same time two velocity components and the diameter, covering a large size range, for each droplet. Insights into the turbulent multiphase flow common in the irrigation problem were derived that allow a comparison between the Reynolds stresses from the DTV and the turbulent kinetic energy from a turbulent Reynolds-averaged Navier-Stokes model. Felis et al. [8] further developed this method by establishing a reproducible study case and by coupling this technique with laser Doppler velocimetry (LDV) and optical probe (OP) techniques.

The latter experimental setup implements a circular nozzle of diameter $d_{n}=1.2 \mathrm{~mm}$ and length $L_{n} / d_{n}=50$, which ensures a fully developed turbulent pipe flow. The nozzle is made of borosilicate glass and the interior wall roughness is considered negligible. A vertical liquid water jet is injected with an average bulk velocity $u_{i n j}=35 \mathrm{~m} / \mathrm{s}$ pointing downward into quiescent air (see Fig. 1). The campaign was made under normal conditions ( $297 \mathrm{~K}$ and $1 \mathrm{~atm})$; the corresponding physical properties are given in Table I. The chosen injection velocity ensures that there is no cavitation in the nozzle. The dimensionless numbers of the case-study conditions are $\operatorname{Re}_{l}=41833, \mathrm{We}_{l}=20158$, $\mathrm{We}_{g}=24.3$, and $\mathrm{Oh}=0.0034$, which makes the jet lie in the second-wind-induced regime detailed above. An estimation of the Taylor-scale Reynolds number at the nozzle exit, based on the results from Ruffin et al. [9], gives $\operatorname{Re}_{\lambda}=400$, typical for fully developed turbulence in monophasic flow. The OP provides the mean liquid mass fraction and volume fraction. A specific LDV apparatus allows us to measure separately the liquid and gas velocities. Finally, a custom DTV algorithm 
TABLE I. Physical properties considered by Felis-Carrasco [10], under normal conditions and in SI units.

\begin{tabular}{llc}
\hline \hline Property & \multicolumn{1}{c}{ Definition } & \multicolumn{1}{c}{ Value } \\
\hline$\rho_{l}$ & water density & $998.3 \mathrm{~kg} / \mathrm{m}^{3}$ \\
$\rho_{g}$ & air density & $1.205 \mathrm{~kg} / \mathrm{m}^{3}$ \\
$v_{l}$ & water kinematic viscosity & $1.004 \times 10^{-6} \mathrm{~m}^{2} / \mathrm{s}$ \\
$v_{g}$ & air kinematic viscosity & $15.11 \times 10^{-6} \mathrm{~m}^{2} / \mathrm{s}$ \\
$\sigma_{l-g}$ & water-air surface tension & $0.073 \mathrm{~N} / \mathrm{m}$ \\
\hline \hline
\end{tabular}

can capture the liquid velocity in the dispersion area of the spray. The DTV measurements are carried out to determine the radial profiles for $x / d_{n} \in[400,800]$ while paying special attention to the depth-of-field estimations to reduce the bias on the droplet-size-velocity correlation. Details are given in [10].

Among the results from this study, it appears that the droplet-size distribution greatly impacts the mean velocity and the Reynolds stress field. This opens the way for a possible segregation of the droplets into several subgroups, as the Reynolds stress tensor shows different behaviors depending on the droplet size. A size class repartition highlights that small droplets tend to show large velocity fluctuations and a Stokes number of $O(1)$, like passive tracers in turbulence, while bigger droplets show a high axial mean velocity with almost zero fluctuations with a Stokes number of $O(100)$, like ballistic objects. However, the statistical analysis is not fine enough to depict precisely any specific group in the overall population.

Furthermore, the question of the drop size and velocity joint distribution remains open for this kind of flow. On the one hand, Villermaux [11] alleged that drop-size distributions are described by a universal $\Gamma$ law, derived from a fine analysis of the ligament dynamics in low-turbulence fragmentation flows. While this law showed good agreement with experiments showing unimodal $[12,13]$ or bimodal size distributions [14], it is legitimate to question its validity in fragmentation flows where turbulence plays a major role. On the other hand, Novikov and Dommermuth [15] used a phenomenological approach based on turbulence to describe the droplet-size distribution. Starting from the idea of similarity, i.e., the cascade process, they proposed a size distribution for turbulent flows based on infinitely divisible distributions [16] and turbulence intermittency [17]. Later on, Rimbert and Sero-Guillaume [18] simplified this approach by considering log-stable distributions, which are easier to handle than infinitely divisible distributions, and Rimbert and Castanet [19] were able to describe the multimodal size distribution produced in a bag-breakup regime with a crossover between Rayleigh-Taylor instability and the turbulent cascading atomization mechanism. Finally, in the context of combustion, Lee and An [20] derived, from the energy balance of a pressure-atomized spray, a quadratic formula for the droplet size as a function of the liquid velocity. Given the short distances over which jets develop in combustion applications, it is natural to wonder about the performance of this formula for agricultural-like configurations.

The present paper focuses on the DTV measurements by Felis et al. [8] and Felis-Carrasco [10]. It offers an analysis of the multimodal size-velocity joint probability distribution function (PDF) and a determination of different subgroups among the overall droplet population. Section II presents the three models cited above. Section III is dedicated to the analysis of the size and velocity distributions and their modeling. Section IV focuses on the determination of the droplet subgroups based on the size-velocity joint PDF and on the investigation of the validity of the quadratic formula proposed by Lee and An [20] for $x / d_{n}$ between 400 and 800.

\section{MODELING THE SIZE DISTRIBUTION OF SPRAY-GENERATED DROPLETS}

In the context of multiphase flows, the governing equations must be derived using a phase indicator $\alpha_{k}$, which indicates the presence of the phase $k$ at any position $\mathbf{x}$ and instant $t$. Assuming 
there is no mass exchange between the phases, the governing equations for a phase $k$ are then [21]

$$
\begin{gathered}
\frac{\partial \rho_{k} \alpha_{k}}{\partial t}+\frac{\partial \rho_{k} \alpha_{k} u_{j, k}}{\partial x_{j}}=0, \\
\frac{\partial \rho_{k} \alpha_{k} u_{i, k}}{\partial t}+\frac{\partial \rho_{k} \alpha_{k} u_{i, k} u_{j, k}}{\partial x_{j}}=\frac{\partial \alpha_{k} \sigma_{i j, k}}{\partial x_{j}}+f_{s, i, k-k^{\prime}} \sigma_{k}^{s},
\end{gathered}
$$

where $u_{j, k}$ stands for the $j$-velocity component of phase $k, f_{s, i, k-k^{\prime}}$ represents the friction force between the phases $k$ and $k^{\prime}$ which applies on the interface $s$ of volumetric density $\sigma_{k}^{s}$, and $\sigma_{i j, k}$ stands for the Cauchy stress tensor associated with phase $k$. Deriving the droplet-size distribution directly from Eqs. (2) is not possible yet and models are needed in order to depict and predict such a distribution. The following sections introduce the different approaches and corresponding droplet-size distributions of Villermaux et al. [22], Novikov and Dommermuth [15], and Lee and An [20].

\section{A. Ligament-mediated spray formation}

In contrast to the classical cascade process in which large elements generate smaller ones, Villermaux et al. [22] proposed an aggregation scenario in which ligaments are the cornerstone. This processus relies on the aggregation kinematics developed by Smoluchowski [23] for solid colloidal particles in Brownian motion. Let $n(v, t)$ be the number of clusters of volume between $v$ and $v+d v$ at the instant $t$. In addition, $N(t)$ is the total number of clusters and $K\left(v, v^{\prime}\right)$ is the frequency of aggregation between clusters of volumes $v$ and $v^{\prime}$. The aggregation kinetics is then governed by

$$
\partial_{t} n(v, t)=-n(v, t) \int_{0}^{\infty} K\left(v, v^{\prime}\right) n\left(v^{\prime}, t\right) d v^{\prime}+\frac{1}{2} \int_{0}^{v} K\left(v^{\prime}, v-v^{\prime}\right) n\left(v^{\prime}, t\right) n\left(v-v^{\prime}, t\right) d v^{\prime} .
$$

When talking about droplets, it is common to assume that the elements are spherical. The element size is then given by the diameter $d$ and one can consider $n(d, t)$ instead of $n(v, t)$. In $[12,22]$ the ligaments were supposed to consist of $v$ independent sublayers resulting from a random particulate motion. Each sublayer consisted of subblobs of size $d^{\prime}$ and their size distribution was denoted by $q\left(d^{\prime}, t\right)$. The interaction between the sizes was assumed to be both random and uncorrelated. According to [24], the evolution of $q\left(d^{\prime}, t\right)$ was governed by a convolution process and the distribution of size $d$ was such that $n(d, t)=N(t) q\left(d^{\prime}, t\right)^{\otimes v}$, where $\otimes$ denoted the convolution product. Equation (3) then becomes

$$
\partial_{t} n(d, t)=-n(d, t) N(t)^{\gamma-1}+\frac{1}{3 \gamma-2} n(d, t)^{\otimes \gamma}
$$

where $\gamma=1+1 / v$, with $v$ the number of ligament layers. Knowing that the process is governed by successive autoconvolutions and that the distribution $q(d, t)$ in each layer is assumed to be independent, the droplet-size distribution $p_{B}$ along the ligament after detachment from the bulk flow is thus described by a $v$ convolution

$$
p_{B}(d)=p_{1}(d)^{\otimes v},
$$

where $p_{1}(d)$ is an elementary distribution corresponding to the size distribution along a ligament layer, once the ligament is detached from the bulk flow. It can be chosen as an exponential distribution characterized by the diameter $\xi_{B}$ of the detached ligament before its breakup [22]. The distribution $p_{B}$ is rewritten as

$$
p_{B}(x=d /\langle d\rangle)=\frac{v^{v}}{\Gamma(v)} x^{(v-1)} e^{-v x},
$$

where $\langle d\rangle=\frac{1}{N(t)} \int d n(d, t) d d$ is the instantaneous mean droplet diameter. Finally, the droplet-size distribution of the spray is given by the composition of the ligament size distribution $p_{L}\left(d_{0}\right)$, with 
$d_{0}$ the size of a ligament, and the droplet-size distribution after the ligament breakup $p_{B}\left(d / d_{0}\right)$,

$$
p(d)=\int_{0}^{\infty} p_{L}\left(d_{0}\right) p_{B}\left(\frac{d}{d_{0}}\right) \frac{d d_{0}}{d_{0}} .
$$

Furthermore, the distribution $p_{L}$ of the ligament size is also $\Gamma$ distributed [25] and Kooij et al. [26] derived the droplet-size distribution of a spray as

$$
p(x=d /\langle d\rangle)=\frac{2(m n)^{(m+n) / 2} x^{(m+n) / 2-1}}{\Gamma(m) \Gamma(n)} \mathcal{K}_{m-n}(2 \sqrt{n m x}),
$$

with $\mathcal{K}_{m-n}$ the modified Bessel function of the second kind. The parameter $m$ sets the order of the ligament size distribution and $n$ the ligament corrugation, previously denoted by $v$.

\section{B. Small-scale intermittency and droplet size}

In his seminal work, Kolmogorov [27,28] stated two main assumptions about isotropic turbulence, which are the scale invariance of eddies and the scale localness of interaction, allowing quantitative predictions for the energy distribution among the scales of the flow. This led to the image of the turbulent process as a cascading process where the turbulent energy injected at a rate $\epsilon$ at larger scales cascades down the smaller scales before being eventually removed due to dissipation, still at a rate $\epsilon$. Following the theoretical remark of Landau and Lifshitz [29], a refinement of the hypothesis on the local structure of turbulence was proposed to take into account the small-scale intermittency of turbulence [30-32]. More details about intermittency are given in [33] and a review of intermittency models is available in [34].

Novikov and Dommermuth [15] proposed a statistical description of droplets in turbulent spray connected with the turbulent dissipation resulting from small-scale intermittency. Similarly to the context of turbulent energy dissipation, the authors proposed that liquid fragments go through a sequential cascade mechanism such that

$$
l \equiv l_{N+1}=l_{1} \prod_{k=1}^{N} b_{k}, \quad b_{k}=\frac{l_{k+1}}{l_{k}} \leqslant 1,
$$

where $l_{1}$ is the initial size of a liquid fragment, $N$ the number of breakups, and $l$ the final size of a droplet at the end of the process. Equation (9) is rewritten as

$$
y \equiv-\ln \left(\frac{l}{l_{1}}\right)=-\sum_{k=1}^{N} \ln \left(b_{k}\right) .
$$

Assuming that the coefficients $b_{k}$ are independent or weakly dependent, if $N$ is large enough, then it follows from the central limit theorem [35] that the distribution of $y$ is normal. Thus the moments of the distribution of $l / l_{1}$ are given by

$$
\left\langle\left(\frac{l}{l_{1}}\right)^{p}\right\rangle=\exp \left(-a p+\sigma^{2} p^{2}\right) .
$$

Note that the right-hand side of Eq. (11) is larger than 1 when $p$ tends towards $+\infty$, which contradicts Eq. (9). Physically speaking, this implies that Eq. (10) breaks the mass conservation. Mathematically speaking, in this situation, the properly normalized characteristic function of the probability function will tend to normal, but not the probability function [15]. Thus, even if the fragmentation process is supposed to be a sequential breakup cascade, the distribution of $l / l_{1}$ is not log-normal.

It follows from the refinement of Kolmogorov hypotheses that the turbulent energy dissipation is not uniformly distributed among the scales. The dissipation average rate over the distance $r$ is denoted by $\epsilon_{r}$. Consider the inertial range of scales $L \gg r \gg l_{*}$, where $L$ is the integral scale and 
$l_{*}$ is an inertial scale which can differ from the Kolmogorov internal scale because of intermittency correction. For three scales $r, \rho$, and $l$ in this range such that $r<\rho<l$, we can introduce the corresponding breakdown coefficients (BDCs)

$$
q_{r, l}=\frac{\epsilon_{r}}{\epsilon_{l}}, \quad q_{r, l} \leqslant \frac{l}{r}, \quad q_{r, l}=q_{r, \rho} q_{\rho, l} .
$$

The scale similarity in a turbulent flow is determined by the following conditions: (i) The probability distribution of BDCs depends only on the ratio of the corresponding scales and (ii) $q_{r, \rho}$ and $q_{\rho, l}$ are statistically independent. From those conditions and Eq. (12) we have, for the moments of the BDCs,

$$
\left\langle q_{r, l}^{p}\right\rangle=\left(\frac{l}{r}\right)^{\mu(p)}, \quad \mu(0)=0,
$$

with the quantity $\mu(p)$ respecting additional properties given in [17]. Note that $\mu(2)=\mu$, with $\mu$ the classic intermittency coefficient [34]. By definition, the characteristic function for $\ln \left(q_{r, l}\right)$ is $\psi(s, l / r)=\left\langle\exp \left[i s \ln \left(q_{r, l}\right)\right]\right\rangle$. By inverting this formula, it could be possible to determine directly the probability distribution of $q_{r, l}$, but it requires costly verifications to ensure the non-negativity of the distribution in order to enforce physical and mathematical meaning [16].

Deriving the distribution of $q_{r, l}$ can be achieved by noting that, for arbitrary ratio $l / r$ and arbitrary integer $n$, Eq. (13) can be written in the form

$$
\psi\left(s, \frac{l}{r}\right)=\psi^{n}\left(s,\left(\frac{l}{r}\right)^{1 / n}\right),
$$

which defines infinitely divisible distribution. The Lévy-Baxter-Shapiro theorem [35] gives the general form of such distributions concentrated on $[0,+\infty[$. Using this theorem allows us to derive a general form of $\mu(p)[16]$,

$$
\mu(p)=\kappa p-\int_{0}^{+\infty} \frac{1-e^{-p x}}{x} F(d x)
$$

where $F$ is a measure on the open interval $\left[0,+\infty\left[\right.\right.$ such that $(1+x)^{-1}$ is integrable with respect to $F$. Knowing $\mu(p)$, it is thus possible to reconstruct the distribution $W$ of $q_{r, l}$ from

$$
W\left(q, \frac{l}{r}\right)=\frac{1}{2 \pi q} \int_{-\infty}^{+\infty} \exp \left[-i s \ln (q)+\mu(i s) \ln \left(\frac{l}{r}\right)\right] d s .
$$

Note that the distribution of $\ln \left(q_{r, l}\right)$ is $Q[\ln (q)]=q W(q)$. Keeping in mind that $r \epsilon_{r} / L \epsilon_{L}$ is analogous to $l / l_{1}$ in Eq. (9), it is possible to use the distribution of $\epsilon_{r} / \epsilon_{l}$ to model the distribution of $l / l_{1}$. Doing so, Novikov and Dommermuth [15] gave one example of a distribution for $y=\ln \left(l / l_{1}\right)$ which only depends on the average $a=\langle y\rangle$ and the standard deviation $\sigma^{2}=\left\langle(y-a)^{2}\right\rangle$ of the population:

$$
p(y)=\frac{a^{3 / 2}}{\sqrt{2 \pi} \sigma y^{3 / 2}} \exp \left\{-\frac{a}{2 \sigma^{2}}\left(a y^{-1 / 2}-y^{1 / 2}\right)^{2}\right\}, \quad y \geqslant 0 .
$$

\section{Integral approach from combustion}

Lee and An [20] followed an integral approach in order to derive a relationship between the droplet diameter and velocity. This approach allowed them to relate the physical quantities at the nozzle exit to the ones downstream, in the jet dispersion zone where atomization is achieved. Consider a control volume $V_{s}$ enveloping the overall spray volume. The argumentation relies on two main assumptions. First, the liquid phase is assumed to achieve the transition from its initial state to the final state of a fully atomized group of spherical droplets within the specified control volume. Second, it is assumed that the viscous dissipation can be written in terms of known parameters such 
as the liquid velocity and dissipation length scale. The integral form of the conservation equations of mass and energy for the liquid phase in the control volume are given by

$$
\begin{aligned}
\rho_{l} u_{i n j} A_{i n j} & =\iint \rho_{l} \frac{\pi d^{3}}{6} u A n \bar{p}(d, u) d d d u \approx n \rho_{l} \frac{\pi}{6} \bar{u} A \sum_{i}^{N} p\left(d_{i}\right) d_{i}^{3} \Delta d_{i}, \\
\rho_{l} \frac{u_{i n j}^{3}}{2} A_{i n j} & =n \rho_{l} \frac{\pi}{12} \bar{u}^{3} A \sum_{i}^{N} p\left(d_{i}\right) d_{i}^{3} \Delta d_{i}+n \bar{u} A \pi \sigma_{l-g} \sum_{i}^{N} p\left(d_{i}\right) d_{i}^{2} \Delta d_{i}+K^{\prime} \mu_{l}\left\langle\left(\frac{\partial u}{\partial y}\right)^{2}\right) V_{s},
\end{aligned}
$$

where $V_{s}$ is the spray volume. The mass conservation is achieved by equating the injected mass flow rate with the mass of the droplets contained in a volume swept by the mean velocity $\bar{u}$ over a spray area $A$. The velocity distribution is simplified to an average drop velocity. The cross-sectional area $A$ represents the physical extent of the spray at a plane where full atomization is achieved and can be calculated from the spray cone angle. The quantities $n, d_{i}, p\left(d_{i}\right)$, and $\Delta d_{i}$ denote the droplet number density, the droplet diameter, the droplet-size distribution, and the droplet-size bin width, respectively. The authors considered that the fragmentation of the jet liquid core into droplets occurs at some velocity scale, taken as the mean liquid velocity $\bar{u}$, and at the length scale of the droplets, taken as the Sauter mean diameter $d_{3,2}$ since it is the scale at which droplets are created. This approach is similar to the classic one of Tennekes and Lumley [36] in which $(\partial u / \partial y)^{2}$ is linked to the Taylor microscale, taken here as $d_{3,2}$. This leads to the estimation of the average viscous dissipation as

$$
\mu_{l}\left\langle\left(\frac{\partial u}{\partial y}\right)^{2}\right\rangle V_{s} \sim \mu_{l}\left(\frac{u}{d_{3,2}}\right)^{2} V_{s} .
$$

Finally, the model has one adjustable parameter $K^{\prime}$, as the exact relationship between the viscous dissipation term and the spray volume is approximated. After solving Eq. (18a) for $n$, substituting Eq. (19) into Eq. (18b) gives a quadratic equation for the $d_{3,2}$-velocity relationship

$$
\rho_{l}\left(\frac{u_{i n j}^{2}-\bar{u}^{2}}{2}\right) d_{3,2}^{2}-6 \sigma_{l-g} d_{3,2}-K \mu_{l} \bar{u}^{2}=0,
$$

where $K$ absorbs the spray volume term for the sake of simplicity. After discarding the negative solution, this leads to a quadratic relationship between $d_{3,2}$ and the velocity

$$
d_{3,2}=\frac{3 \sigma_{l-g}+\sqrt{9 \sigma_{l-g}^{2}+K \rho_{l} \mu_{l} \bar{u}^{2} \frac{u_{i n j}^{2}-\bar{u}^{2}}{2}}}{\rho_{l} \frac{u_{i n j}^{2}-\bar{u}^{2}}{2}} .
$$

Equation (21) shows good agreement with the literature for pressure-atomized sprays with and without swirl and allow Lee and An [20] to reconstruct the droplet-size distribution from the droplet velocity distribution.

\section{BIMODAL SIZE AND VELOCITY DISTRIBUTIONS}

This section first gives a statistical description and the number PDF of the data from Felis-Carrasco [10] and then tackles their modeling in the framework of turbulence. The DTV measurements were obtained from positions along the jet axis located at $x / d_{n}=$ $\{400,500,600,700,800\}$. At each axial position, measurements were made at different positions perpendicular to the jet. In order to capture all the spray development, the limit radial positions are different between the axial positions. At $x / d_{n}=400$, the radial positions span from $y / d_{n}=-20$ to $y / d_{n}=20$ and they span from $y / d_{n}=-32$ to $y / d n=32$ at $x / d_{n}=800$. The measurements give access to the diameter $d$, the axial velocity $u$, and the radial velocity $v$ of every droplet over a large section of the dispersion zone. Technically, the droplets are assumed to be spheroids and 
TABLE II. Mean values of the distributions of $d, u$, and $v$.

\begin{tabular}{lccccc}
\hline \hline$x / d_{n}$ & 400 & 500 & 600 & 700 & 800 \\
\hline$\langle d\rangle(\mu \mathrm{m})$ & 96.82 & 91.22 & 95.60 & 93.63 & 97.89 \\
$\langle d\rangle_{\mathcal{V}}(\mu \mathrm{m})$ & 125.0 & 126.2 & 129.3 & 125.0 & 121.7 \\
$\langle\cdot\rangle_{\mathcal{V}} /\langle\cdot\rangle$ & 1.29 & 1.38 & 1.35 & 1.33 & 1.24 \\
$\langle u\rangle_{(\mathrm{m} / \mathrm{s})}$ & 12.48 & 13.20 & 12.97 & 12.01 & 12.00 \\
$\langle u\rangle_{\mathcal{V}}(\mathrm{m} / \mathrm{s})$ & 21.53 & 20.35 & 19.78 & 18.17 & 16.72 \\
$\langle\cdot\rangle_{\mathcal{V}} /\langle\cdot\rangle$ & 1.72 & 1.54 & 1.52 & 1.51 & 1.39 \\
$\langle v\rangle_{(\mathrm{mm} / \mathrm{s})}$ & 2.1 & -38.9 & -16.7 & -40.3 & -2.9 \\
$\langle v\rangle_{\mathcal{V}}(\mathrm{mm} / \mathrm{s})$ & -161.5 & -210.4 & -84.4 & -129.6 & -54.3 \\
$\langle\cdot\rangle_{\mathcal{V}} /\langle\cdot\rangle$ & -76.9 & 5.41 & 5.05 & 32.2 & 18.7 \\
\hline \hline
\end{tabular}

the droplet volume $V$ is estimated from the semiaxes given by the DTV measurements. Then the droplet diameter $d$ is calculated as the diameter of the sphere of the same volume. Unless otherwise mentioned, the statistics given at a position $x / d_{n}$ aggregate all the data over the positions $y / d_{n}$. For the sake of clarity, the number PDF of any variable $\zeta$ will be denoted by $\mathcal{P}_{\zeta}$ in the following.

\section{A. Experimental distributions}

As a first step, one could look at the mean values of the velocity distributions and the size distribution. Table II gives the algebraic mean $\langle\cdot\rangle$, the volume weighted mean $\langle\cdot\rangle_{\mathcal{V}}$, and the ratio of the two for each distribution and each axial position. For any variable $\zeta$, the volume-weighted average reads $\langle\zeta\rangle_{\mathcal{V}}=\sum_{i=1}^{N_{\text {tot }}} V_{i} \zeta_{i} / \sum_{i=1}^{N_{\text {tot }}} V_{i}$, where $i$ is the droplet index, $N_{\text {tot }}$ the total number of droplets, $V_{i}$ the volume, and $\zeta_{i}$ the $\zeta$ value of the $i$ th droplet. While the ergodic condition can often be assumed, it is important to note here that both means systematically depart from each other for the three distributions. For the distribution of $d$, the volume-weighted mean is at least $24 \%$ larger than the algebraic mean, while this difference decreases from $72 \%$ to $39 \%$ for the distribution of $u$. Note as well that the means of $u$ decrease along $x / d_{n}$. Concerning the distribution of $v$, the ratio of the two means shows a consequent variability, due to the proximity to zero of the mean values. Even if the variability is large, the means show at least one order of magnitude of difference. In the prospect of working with properly normalized variables, this systematic departure has to be accounted for in the choice of the mean to use for the normalizing procedure. The mass and energy conservation equations for the two-phase jet, introduced in Sec. II, rely on the phase indicator $\alpha_{k}$. To ensure mass conservation in this context, the average of a variable $\zeta$ in the phase $k$ must be expressed as $\left\langle\alpha_{k} \zeta\right\rangle$, which practically is equivalent to the volume-weighted mean. Thus, we choose the volume-weighted mean $\langle\zeta\rangle_{\mathcal{V}}$ as the normalizing quantity of $\zeta$. Doing so is equivalent to normalizing the droplet diameter by $d_{4,3}$ and the velocities by the bulk velocity of the dispersion phase. In the following, normalization is achieved by using the mean weighted by the droplet volume, except for the radial velocity $v$. Because $\langle v\rangle_{\mathcal{V}}$ is close to 0 and could be misevaluated from the experimental measurements, the radial velocity $v$ is normalized by $\langle u\rangle_{\mathcal{V}}$.

Knowing how to properly normalize the present data, it is possible to compute the PDF of $d /\langle d\rangle_{\mathcal{V}}, u /\langle u\rangle_{\mathcal{V}}$, and $v /\langle u\rangle_{\mathcal{V}}$ (see Fig. 2). The size distribution is computed over 1730 bins and the velocity distributions over 80 bins, with a total number of occurrences close to 400000 on average. In addition to the mean, the three distributions are characterized by higher-order statistical moments. Figure 3 gives the evolution of the statistical moments up to the order 4 over the available $x / d_{n}$ positions. The high-order moments under consideration here are the standard deviation $\sigma$, the skewness $S$, and the kurtosis $\kappa$, also referred to as flatness.

The size distribution $\mathcal{P}_{d /\langle d\rangle_{\mathcal{V}}}$ presents an important skewness and a very large kurtosis. The former decreases along $x$ from 10 to 5 and the latter decreases from 155 to roughly 35 . Such values of skewness represent the fact that the distribution spans two decades $d /\langle d\rangle_{\mathcal{V}} \in[0.1,40]$ and that 

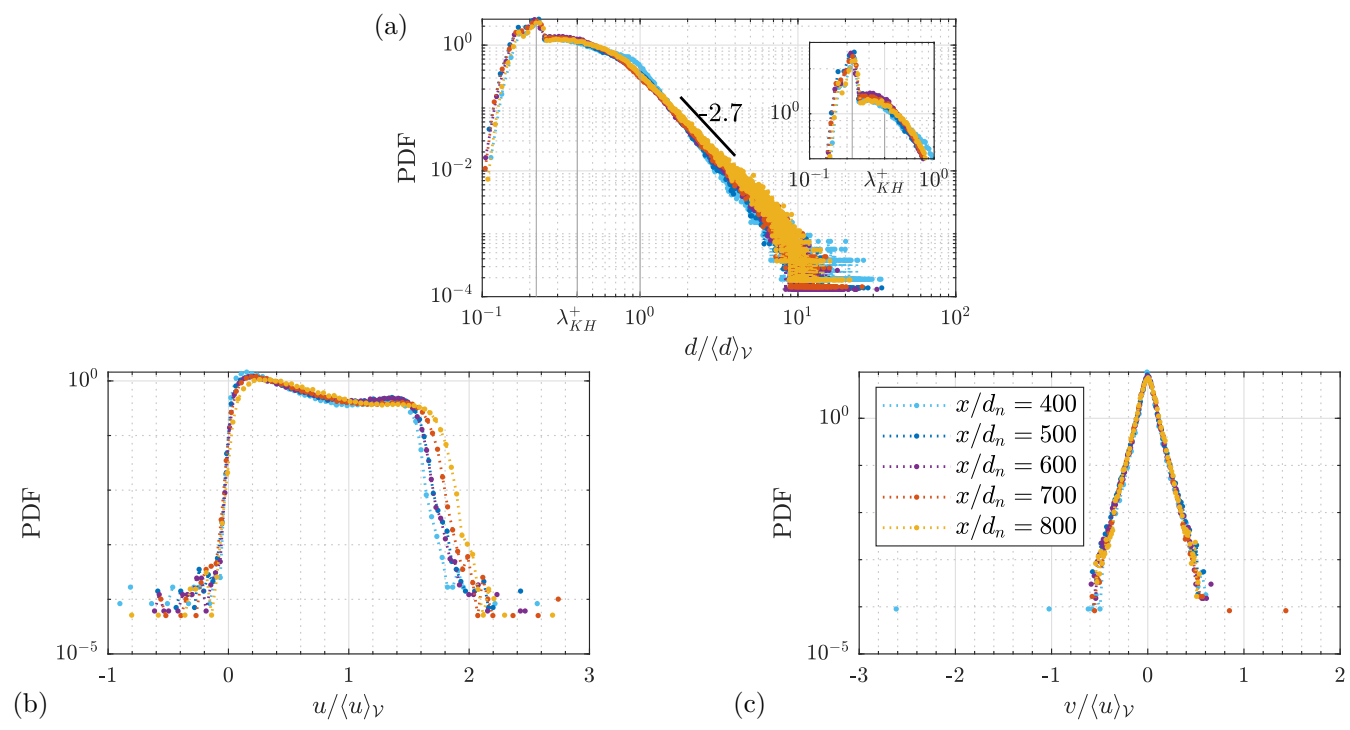

FIG. 2. Distributions of (a) $d /\langle d\rangle_{\mathcal{V}}$, (b) $u /\langle u\rangle_{\mathcal{V}}$, and (c) $v /\langle u\rangle_{\mathcal{V}}$ for experimental data provided by FelisCarrasco [10]. (a) A logarithmic scale and (b), (c) a semilogarithmic scale are used.

most of the droplets lie in the first decade, with the median value of $d /\langle d\rangle_{\mathcal{V}}$ being equal to 0.51 on average. Regarding the kurtosis values, they are representative of the presence of large values, relative to the mean, in the tail of the distribution. Finally, the value of the standard deviation is almost constant over the five axial positions and equal to 0.93 on average, typical of distributions showing a region with concentrated data. Indeed, on average, the $25 \%$ and $75 \%$ quartiles are equal to 0.29 and 0.86 and the average interquartile is then 0.57 , a range in which $50 \%$ of the droplets lie. Concerning the tail behavior, the distributions show a power-law decay scaling as $d /\langle d\rangle_{\mathcal{V}}^{-2.7}$,

\section{(a)}
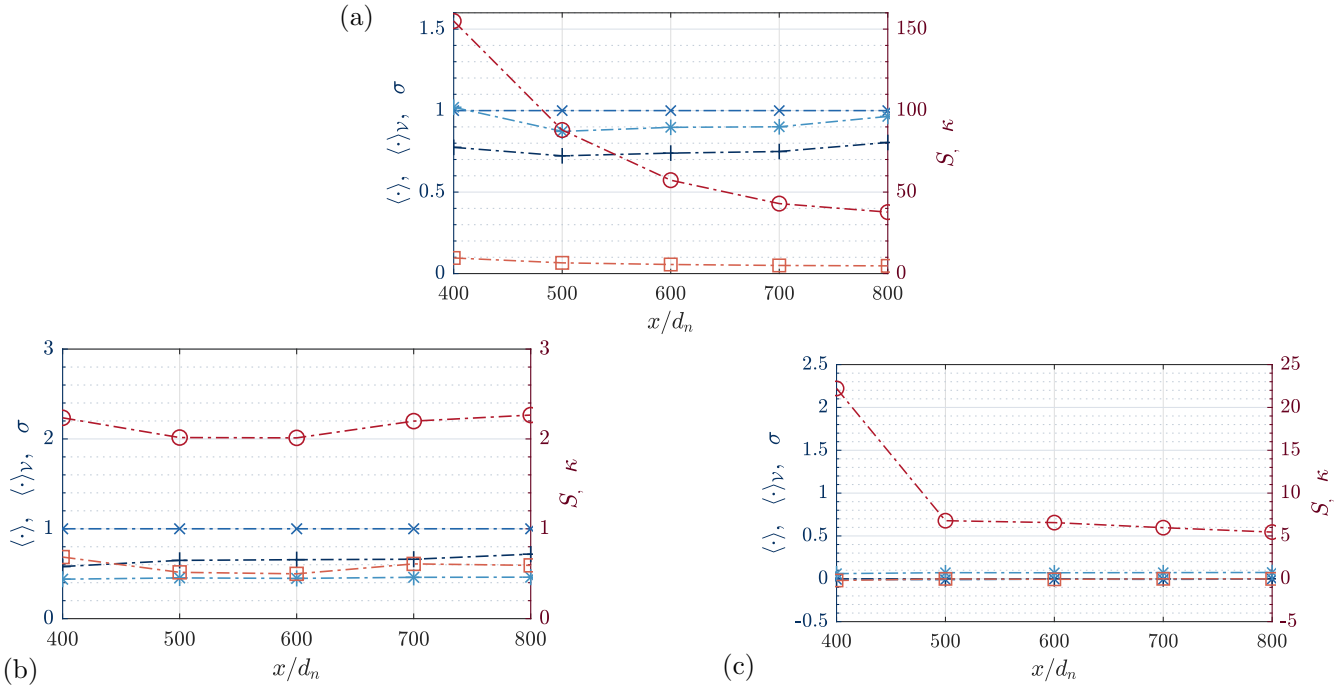

FIG. 3. Statistical moments of (a) $d /\langle d\rangle_{\mathcal{V}}$, (b) $u /\langle u\rangle_{\mathcal{V}}$, and (c) $v /\langle u\rangle_{\mathcal{V}}$ for experimental data provided by Felis-Carrasco [10]. The blue lines represent $\langle\cdot\rangle(+),\langle\cdot\rangle_{\mathcal{V}}(\times)$, and $\sigma(*)$. The red lines represent $S(\square)$ and $\kappa$ (०). 
which departs from the exponential decay found by Simmons [37]. Furthermore, two modes can be seen on the distribution at each $x / d_{n}$ : one for $d /\langle d\rangle_{\mathcal{V}} \approx 0.2$ and the other for $d /\langle d\rangle_{\mathcal{V}} \approx 0.4$. At the location $x / d_{n}=400$, a third mode is also visible for $d /\langle d\rangle_{\mathcal{V}} \approx 1.0$. Here it is worth noting that the second mode corresponds to the characteristic length scale of the Kelvin-Helmholtz instability [38]: $\lambda_{\mathrm{KH}}=\sigma / \rho_{g} u_{i n j}^{2}=49.6 \mu \mathrm{m}$. Once normalized, this length scale is denoted by $\lambda_{\mathrm{KH}}^{+}$ and $\lambda_{\mathrm{KH}}^{+} \approx 0.4$. We recall that $\lambda_{\mathrm{KH}}$ also respects $\mathrm{We}_{g}=d_{n} / \lambda_{\mathrm{KH}}$. Finally, close similarity is achieved for the distributions between $x / d_{n}=500$ and $x / d_{n}=800$ and characterizes a converged behavior with steady mechanisms in this range.

The distribution $\mathcal{P}_{u /\langle u\rangle_{\mathcal{V}}}$ presents two modes for every $x / d_{n}$ position as well. They are located around $u /\langle u\rangle_{\mathcal{V}} \approx 0.2$ and 1.4. In contrast to $\mathcal{P}_{d /\langle d\rangle_{\mathcal{V}}}$, this distribution presents a slight departure between the tails on the right side and does not show any additional mode at $x / d_{n}=400$. Except for this modest departure, the similarity is close for the five axial positions. Concerning the statistical moments, this PDF is characterized by lower skewness and kurtosis values than previously. Both are almost constant and on average are equal to 0.58 and 2.14, respectively. This is representative of the fact that the distribution shows a minor asymmetry, due to a difference of predominance between the two modes, and that the distribution tails are short. The limited spanning of the distribution over $u /\langle u\rangle_{\mathcal{V}}$ can be understood as a preponderant effect of the boundary conditions. The right limit might be enforced by the fact that the droplet velocity reaches a maximum on the centerline, close to the mean liquid velocity on the jet axis. The left limit might be enforced by the overall advection of the liquid phase towards increasing $x / d_{n}$, which prevent droplets from reaching negative values for $u$, i.e., moving back to the nozzle. As for the skewness and the kurtosis, the standard deviation of the distribution is almost constant and equal to 0.45 , characteristic once again of the data concentration. On average, the $25 \%$ and $75 \%$ quartiles are equal to 0.27 and 1.00 , and thus the average interquartile is 0.73 . Finally, the values of $S$ and $\kappa$ depart from the ones obtained in the case of a monophasic jet. The distribution of axial velocity for such jet is characterized by a skewness of -0.5 and a flatness of 2.8, which is almost Gaussian. Here the present $u$ distribution shows a reverse asymmetry and a shorter spanning.

The distribution $\mathcal{P}_{v /\langle u\rangle_{\mathcal{V}}}$ presents only one maximum located at 0 . Its skewness is -0.05 on average and the distribution can be considered symmetric. The distribution kurtosis seems to tend to 5 but is equal to 9.39 on average as its value at $x / d_{n}=400$ is relatively large, which is characteristic of a large tail span and a strong departure from Gaussianity. Finally, the standard deviation is almost constant over the axial positions with an averaged value of 0.069 , which shows a constant distribution width along $x$. This distribution seems to behave in a more classic manner than the distribution of $u /\langle u\rangle_{\mathcal{V}}$ and $d /\langle d\rangle_{\mathcal{V}}$ as it shows only one mode and characteristics similar to what can be found in the turbulence literature.

\section{B. Modeling}

Multiphase flows are inherently multidimensional. Their multidimensionality originates not only in the physical space but also in the phase space. Typically, every droplet of the present jet flow is, at first sight, characterized by three parameters: its size and two velocity components. Thus, in order to depict this population, one would need to propose a model able to capture the behavior of a three-dimensional joint PDF over the available axial positions. Doing so is very complex and it is easier to first have a closer look at the PDF of each parameter. This section proposes a model for the droplet-size distribution and the axial-velocity distribution.

\section{Drop-size distribution}

Sections II A and II B introduce two models of droplet distributions derived from different backgrounds. The first one, given by Eq. (8), is derived from a fine analysis of the ligament mechanics [22] experimentally studied in configurations like impacting droplets [25] or coaxial jet [12], close to the nozzle. The second one, given by Eq. (17), is derived from a phenomenological approach taking place in the framework of intermittent turbulence [16] and was initially developed 

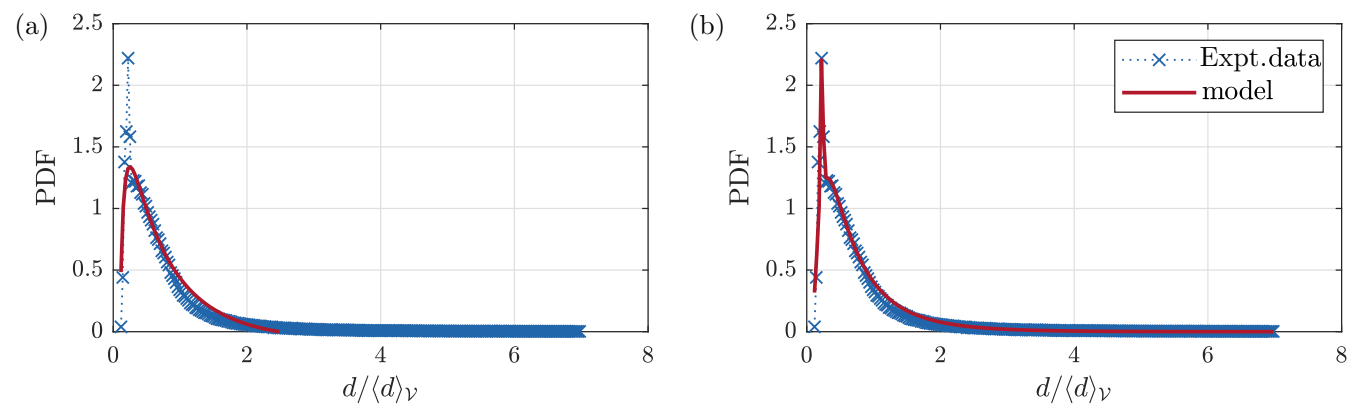

FIG. 4. Fit of the marginal distribution of $d /\langle d\rangle_{\mathcal{V}}$ at the axial position $x / d_{n}=800$ by the distributions from (a) Kooij et al. [26] and (b) Novikov and Dommermuth [15].

for turbulent spray around ships [15]. Those distributions will be respectively denoted by $\operatorname{Prob}_{\Gamma}$ and $\operatorname{Prob}_{\epsilon}$ in this section.

A test campaign was carried out to compare the performance of each distribution to model the bimodal distribution obtained in Fig. 2. In order to reproduce the two modes of the experimental distribution, the fitting functions are defined as a linear combination of two reference distributions such that

$$
\begin{gathered}
f_{\Gamma}(x)=\alpha_{1} \operatorname{Prob}_{\Gamma}\left(x, m_{1}, n_{1}\right)+\alpha_{2} \operatorname{Prob}_{\Gamma}\left(x, m_{2}, n_{2}\right), \\
f_{\epsilon}(x)=\alpha_{1} \operatorname{Prob}_{\epsilon}\left(x, a_{1}, \sigma_{1}\right)+\alpha_{2} \operatorname{Prob}_{\epsilon}\left(x, a_{2}, \sigma_{2}\right),
\end{gathered}
$$

where $\alpha_{i}, m_{i}, n_{i}, a_{i}$, and $\sigma_{i}$ are the fitting parameters. Note that both fit functions present the same number of fit parameters. The fitting algorithm used is the one of the EZYFIT toolbox developed by Moisy on MATLAB [39]. This algorithm is said to be able to capture a reference signal if the set of initial guesses is of the order of the set of converged parameters.

A fit is said to show good agreement with a given reference when the Pearson correlation coefficient, denoted by $r$, is close to 1 . For a more discriminating criterion, one can use $r^{2}$. The performance of the fitting functions to capture the experimental distribution is measured with $r^{2}$. Both distributions are tested over 19 different initial guesses. The focus is on the region showing the two experimental modes, while the tail of the distribution is omitted. Thus the fitting procedure uses as reference the experimental distribution truncated at $d /\langle d\rangle_{\mathcal{V}}=7$ and the fitting is computed in the linear mode. Figure 4 gives the best results obtained from this campaign for each fitting function. The initial guesses, the final parameter values, and $r^{2}$ are given in Table III. The main difference between the two fitting functions is their ability to capture both peaks of the distribution. Using the algorithm from the EZYFIT toolbox, $f_{\Gamma}$ systematically fails to capture the mode at $d /\langle d\rangle_{\mathcal{V}} \approx 0.2$,

TABLE III. Initial guesses, final parameters, and $r^{2}$ for (a) Eq. (22a) and (b) Eq. (22b) given in Fig. 4. The parameter values are truncated at the third decimal.

(a)

\begin{tabular}{lccccccc}
$f_{\Gamma}$ & $\alpha_{1}$ & $m_{1}$ & $n_{1}$ & $\alpha_{2}$ & $m_{2}$ & $n_{2}$ & $r^{2}$ \\
\hline initial & 1 & 1 & 1 & 0.9 & 0.9 & 0.9 & \\
final & 5.26 & 1.04 & 1.20 & -10.86 & 0.10 & 11.78 & 1.04 \\
$f_{\epsilon}$ & $\alpha_{1}$ & $a_{1}$ & $\sigma_{1}$ & $\alpha_{2}$ & $a_{2}$ & $\sigma_{2}$ & $r^{2}$ \\
\hline initial & 1 & 1 & 1 & 0.5 & 0.5 & 0.5 & \\
final & 0.99 & 0.79 & 0.66 & 0.05 & 0.22 & 0.01 & 0.97 \\
\hline \hline
\end{tabular}



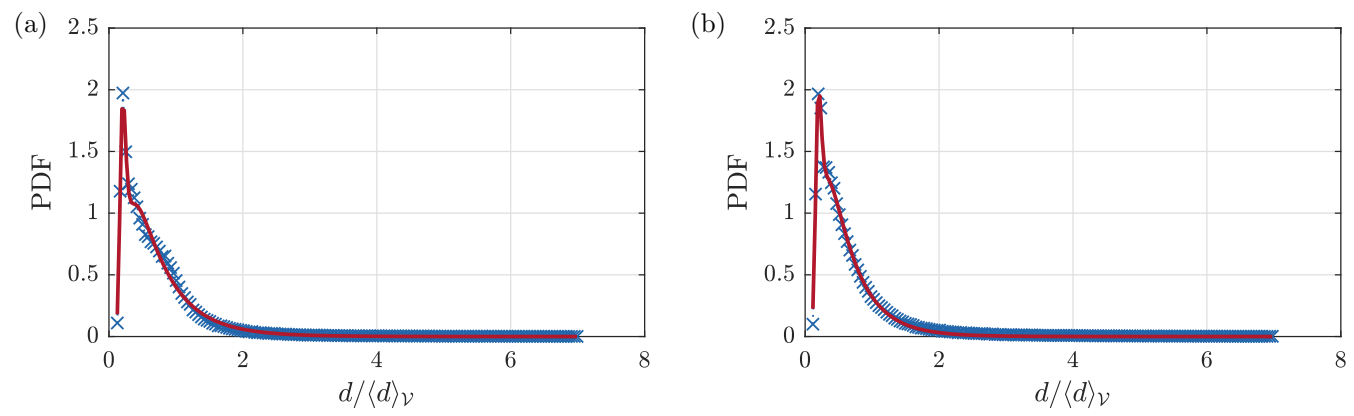

FIG. 5. Fit of the marginal distributions of $d /\langle d\rangle_{\mathcal{V}}$ at the axial position (a) $x / d_{n}=400$ and (b) $x / d_{n}=600$ by the droplet-size distribution from Novikov and Dommermuth [15].

while $f_{\epsilon}$ is able to capture it for one set of initial guesses. One of the two amplitudes for the fitting $f_{\Gamma}$ is negative. The fit is then not physical, as the objective is to model each mode with one distribution. This behavior is observed for a large part of the chosen sets of initial guesses. It could result from the optimization procedure for which a configuration with two modes does not perform as well as the one in Fig. 4. Improvements of the fitting algorithm could be done to implement, for example, parameter constraint or point weighting, to balance the weight of the points regarding their experimental importance. So far, under previous considerations and limits, the fitting function $f_{\epsilon}$ shows a better performance than $f_{\Gamma}$.

In order to test the reproducibility of this result, the function (22b) is tested over the four other $x / d_{n}$ positions using the set of final parameters given in Table III as the initial guess. The fits for the positions $x / d_{n}=400$ and 600 are given in Fig. 5. Their respective Pearson correlation coefficient values are 0.96687 and 1.0026. In both cases, the two modes located at $d /\langle d\rangle_{\mathcal{V}} \approx 0.2$ and 0.4 are captured. The third mode of the distribution for $x / d_{n}=400$ is not captured, which can be expected as $f_{\epsilon}$ is the combination of two distributions $\operatorname{Prob}_{\epsilon}$. The distributions for $x / d_{n} \in\{500,700\}$ show behavior similar to the one at $x / d_{n}=600$, which is consistent with the distribution similarity observed for $x / d_{n} \geqslant 500$.

The distribution derived by Novikov and Dommermuth [15] seems to model well the present experimental distribution, obtained from a turbulent flow, particularly for capturing both modes located at small $d /\langle d\rangle_{\mathcal{V}}$. Conversely, under the limitations of the present campaign scope and methodology, the distribution from Kooij et al. [26] did not capture the higher and thinner peak of the experimental distribution.

\section{Axial-velocity distribution}

Similarly to the size distribution, the axial-velocity distribution shows two distinct modes. Looking at the distributions $\mathcal{P}_{u /\langle u\rangle_{\mathcal{V}}}$ for different $y / d_{n}$ positions, Fig. 6 reveals that only the mode for $u /\langle u\rangle_{\mathcal{V}} \approx 0.2$ remains in the dispersion zone of the jet, while the mode for $u /\langle u\rangle_{\mathcal{V}} \approx 1.4$ has importance only in the region close to the jet axis, i.e., for $y / d_{n}= \pm 8$. In addition, the distributions over the radial positions show symmetry with respect to the jet axis. The exploration of modeling is thus carried out differently in this section. The focus is first on modeling the axial-velocity distribution in the dispersion region of the jet. We choose the position $y / d_{n}=20$ as it is the position farther away from the jet axis which is available for all $x / d_{n}$ positions. The exploration is performed by testing the distributions considered in the study of Yoon [40] on the effects of the Weber number on the droplet-size distribution in a turbulent flow developing up to $x / d_{n}=24$. Then the insights from this step are used to model the velocity mode $u /\langle u\rangle_{\mathcal{V}} \approx 0.2$, while another distribution is proposed for the second mode, in order to depict the overall axial-velocity distribution. 

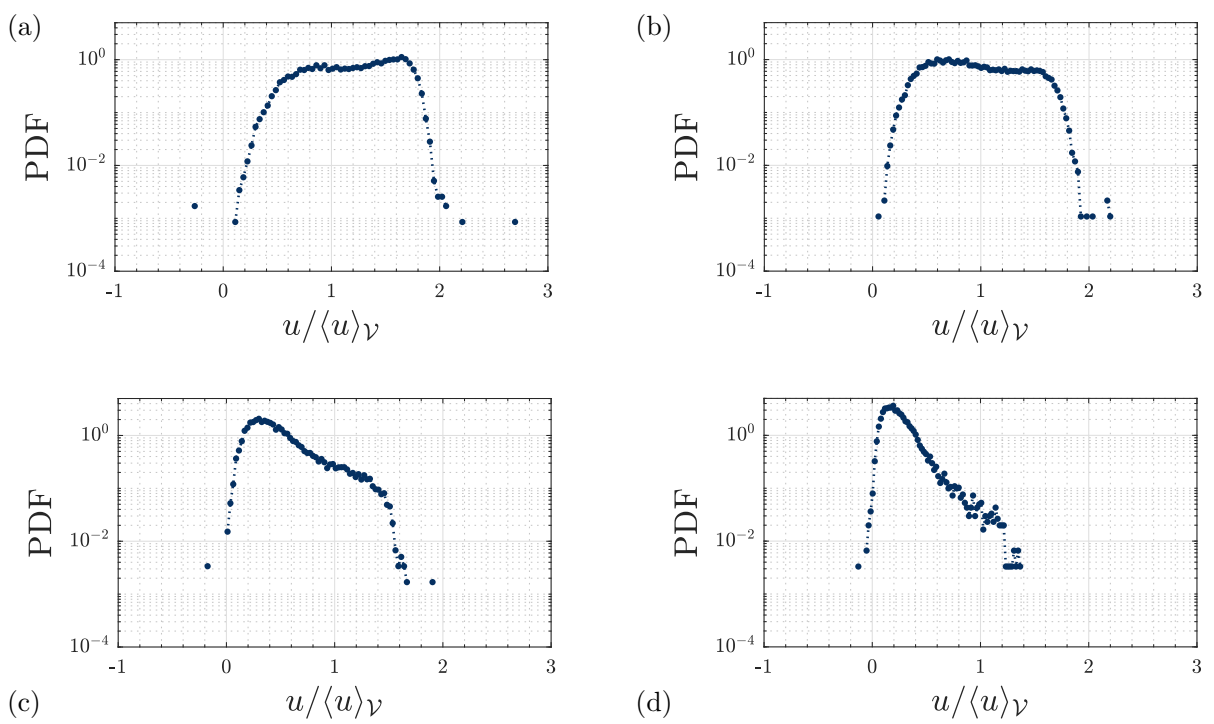

FIG. 6. Distributions of $u /\langle u\rangle_{\mathcal{V}}$ for $x / d_{n}=800$ at the radial positions (a) $y / d_{n}=0$, (b) $y / d_{n}=-8$, (c) $y / d_{n}=-20$, and (d) $y / d_{n}=-32$.

In the aforementioned study, Yoon [40] considers three different PDFs to model the droplet velocity distributions: the Rosin-Rammler distribution $f_{\mathrm{RR}}$, the log-normal distribution $f_{\mathrm{LN}}$, and the Nukiyama-Tanasawa distribution $f_{\mathrm{NT}}$. They are respectively defined by

$$
\begin{aligned}
& f_{\mathrm{RR}}(u)=\frac{q X^{q-1}}{X^{q}} \exp \left[-\left(\frac{u}{X}\right)^{q}\right], \\
& f_{\mathrm{LN}}(u)=\frac{1}{\sqrt{2 \pi} \sigma u} \exp \left(-\frac{[\ln (u)-\mu]^{2}}{2 \sigma^{2}}\right), \\
& f_{\mathrm{NT}}(u)=a \times u^{p} \exp \left(-b u^{c}\right)
\end{aligned}
$$

and show good agreement with experimental and numerical data for a round jet injected in quiescent air at high liquid Weber number, in the so-called atomization regime [1].

In order to model the axial-velocity distribution in the dispersion zone of the jet, the three distributions of Eq. (23) are tested at the radial position $y / d_{n}=20$ over the five axial positions. To ensure the collapse of the PDF, the data have to be normalized by the averaged velocity at $y / d_{n}=20$, denoted by $\langle u\rangle_{\mathcal{V}}^{y / d_{n}=20}$. The fitting procedure is carried out in both the linear mode and logarithmic mode. Fitting with the log-normal distribution or the Nukiyama-Tanasawa distribution offers accurate results on the first try with initial guesses set as unity. However, several sets of initial guesses have to be tried for the Rosin-Rammler distribution to explore the performance of the fitting function.

The discriminating criterion used here is the average of $r^{2}$ over the $x / d_{n}$ positions. The fitting function offering the $r^{2}$ value the closest to unity, on average, is the log-normal distribution $f_{\mathrm{LN}}$ computed in the linear mode. The fitting procedure gives $\mu=0.78$ and $\sigma=0.75$ as final parameter values. Over the five $x / d_{n}$ positions, the correlation coefficient is such that $\left|r^{2}-1\right| \in[0.03,0.28]$ and the mean $r^{2}$ is equal to 0.97 . The upper bound of $\left|r^{2}-1\right|$ is obtained for $x / d_{n}=400$. The fitting of the experimental data by $f_{\mathrm{LN}}$ is given in Fig. 7 .

When looking closely at the distribution of $u /\langle u\rangle_{\mathcal{V}}$ over the $y / d_{n}$ positions, it appears that only the mode for $u /\langle u\rangle_{\mathcal{V}} \approx 0.2$ remains in the dispersion zone of the jet. The previous tests show that the $\log$-normal distribution fits well the experimental data at $y / d_{n}=20$. In order to fit the distribution 

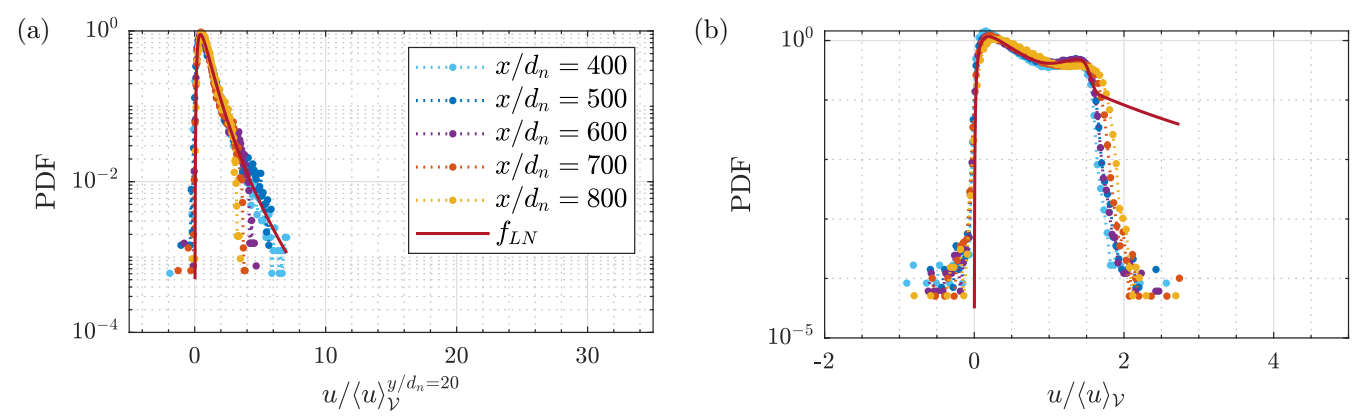

FIG. 7. (a) Fit of the distribution of $u /\langle u\rangle_{\mathcal{V}}^{y / d_{n}=20}$ at the radial position $y / d_{n}=20$ by a log-normal distribution (23b) and (b) fit of the distribution $\mathcal{P}_{u /\langle u\rangle \mathcal{V}}$ over all the radial positions by the model $f(u)$. The dotted lines represent the experimental data and the solid line represents the models. Both graphs use a semilogarithmic scale.

of $u /\langle u\rangle_{\mathcal{V}}$ for each $x / d_{n}$ position, as it was performed in the preceding section, it is possible to build a fitting function as a linear combination of the two distributions. Knowing the performance of $f_{\mathrm{LN}}$ to fit the mode $u /\langle u\rangle_{\mathcal{V}} \approx 0.2$ in the dispersion region, we choose one of the two distributions to be a $\log$-normal distribution with $\mu=0.78$ and $\sigma=0.75$. To be able to reproduce the right asymmetry of the mode for $u /\langle u\rangle_{\mathcal{V}} \approx 1.4$, the second distribution is chosen to be a skewed normal distribution. This distribution is denoted by $f_{\mathrm{SN}}$ and is defined by

$$
f_{\mathrm{SN}}=\frac{1}{\sqrt{2 \pi} \sigma} e^{-[(x-\mu) / \sqrt{2} \sigma]^{2}}\left[1+\operatorname{erf}\left(S \frac{x-\mu}{\sqrt{2} \sigma}\right)\right],
$$

where $\mu, \sigma$, and $S$ are the mean, the standard deviation, and the skewness of the distribution, respectively. The fitting function is then

$$
f(u)=\alpha_{1} f_{\mathrm{LN}}(u)+\alpha_{2} f_{\mathrm{SN}}(u)
$$

The fit of the experimental data over all the radial positions by Eq. (25) is given in Fig. 7. As expected, the mode for $u /\langle u\rangle_{\mathcal{V}} \approx 0.2$ is well captured by $f$ and the fitting function reproduces the left boundary for $u /\langle u\rangle_{\mathcal{V}}=0$. Regarding the mode for $u /\langle u\rangle_{\mathcal{V}} \approx 1.4$, the fitting function is able to capture the peak but fails to reproduce the right boundary for $u /\langle u\rangle_{\mathcal{V}} \geqslant 1.5$. The overestimation of the distribution tail towards $+\infty$ is due to the participation of the log-normal distribution which does not decay fast enough. As a consequence, the fitting function $f$ correctly captures the experimental axial velocity only over the range $u /\langle u\rangle_{\mathcal{V}} \in[0,1.50]$. As a reminder, the average interquartile range of $\mathcal{P}_{u /\langle u\rangle_{\mathcal{V}}}$, given in Sec. III A, is $u /\langle u\rangle_{\mathcal{V}} \in[0.27,1.00]$. The validity range of $f$ not is only larger than the average interquartile range but also contains it. So the fitting function $f$ accurately depicts more than $50 \%$ of the droplet population.

Using the ability of the log-normal distribution to describe accurately the axial-velocity PDF in the dispersion region of the jet, a fitting function was built up as a linear combination of log-normal and skewed normal distributions to depict $\mathcal{P}_{u /\langle u\rangle_{\mathcal{V}}}$ over all the radial positions. This fitting function captures both modes and accurately depicts more than $50 \%$ of the droplet population but fails to depict the distribution tail towards $+\infty$ and so the right boundary on the axial velocity.

\section{DROPLET-SIZE-VELOCITY JOINT DISTRIBUTION AND POPULATION SUBGROUPS}

The presence of bimodal distributions for the droplet size and axial velocity was highlighted in the preceding section. The modes of each distribution could naturally represent a subgroup of droplets characterized by a given size or axial-velocity range. Thus, the present droplet population would present two subgroups with distinct velocities, $u /\langle u\rangle_{\mathcal{V}}$ around 0.2 and 1.4, and two other subgroups with specific sizes, $d /\langle d\rangle_{\mathcal{V}}$ around to 0.2 and 0.3 (see Fig. 2). However, the PDFs of 

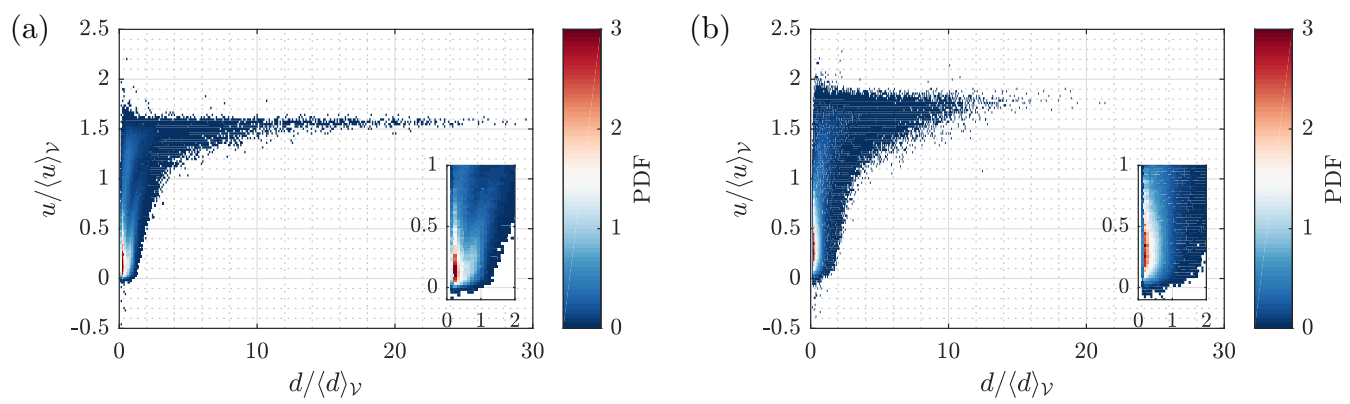

FIG. 8. Droplet-size-velocity joint PDF at (a) $x / d_{n}=400$ and (b) $x / d_{n}=800$.

$u /\langle u\rangle_{\mathcal{V}}$ and $d /\langle d\rangle_{\mathcal{V}}$ do not give any information about the correlation between the modes and thus the subgroups. In Sec. IV A the focus is on the droplet-size-velocity joint PDF to determine droplet subgroups. The validity of the quadratic formula given by Lee and An [20] to model the joint PDF for $x / d_{n} \geqslant 400$ is investigated in Sec. IV B.

\section{A. Characterization of size-velocity subgroups}

In a previous study, Felis et al. [8] highlighted the existence of different droplet behaviors depending on the droplet size and velocity. The Reynolds stress field, computed for different size classes, arbitrarily set, emphasizes that small droplets tend to show large velocity fluctuations while bigger droplets show a high axial mean velocity with almost zero fluctuations. The distributions of the droplet size and axial velocity presented in Sec. III show several modes, indicating the potential existence of droplet subgroups within the overall population. However, no information regarding the mode correlation is yet available. The influence of the size on the axial velocity and vice versa is given by the joint PDF of the droplet size and axial velocity, presented in Fig. 8 for the axial positions $x / d_{n}=400$ and 800 .

First and foremost, a comment must be made on the joint PDF values being larger than one. Those quantities are computed with a regular sampling such that the axis along $d /\langle d\rangle_{\mathcal{V}}$ is sampled in 340 sets and the axis along $u /\langle u\rangle_{\mathcal{V}}$ in 150 sets. Consider the joint PDF at $x / d_{n}=400$. Each axis respectively spans [0.10,33.59] and [-0.92, 2.58]. Thus the bin widths along $d /\langle d\rangle_{\mathcal{V}}$ and $u /\langle u\rangle_{\mathcal{V}}$ are equal to $\Delta_{x}=9.85 \times 10^{-2}$ and $\Delta_{y}=2.33 \times 10^{-2}$, respectively. In a given bin, the value of the joint PDF is calculated as the product of the probability in this bin and the inverse of the bin area. As probabilities are truly between 0 and 1 , the PDF values lie between 0 and $\left(\Delta_{x} \Delta_{y}\right)^{-1}=4.36 \times 10^{2}$. For $x / d_{n}=400$, the maximum value of the joint probability is $7.9 \times 10^{-3}$. Then it is natural that the joint PDF values in Fig. 8 span up to around 3.

Both joint PDFs present a limited extension along the axis $u /\langle u\rangle_{\mathcal{V}}$, included within $[-0.1,2]$, while the extension along $d /\langle d\rangle_{\mathcal{V}}$ is limited on 0 but spans towards large positive values, up to 30 for $x / d_{n}=400$. This characterizes a data set with little dispersion along the velocity axis and important dispersion along the size axis, which corresponds to the behavior of the PDF of $u /\langle u\rangle_{\mathcal{V}}$ and the one of $d /\langle d\rangle_{\mathcal{V}}$. The maximum joint PDF values are concentrated in a relatively limited region located at small sizes and low axial velocities. The tail expansion along $d /\langle d\rangle_{\mathcal{V}}$ is specific in the sense that it exists only for high axial velocities.

For both axial locations, the joint PDF tail tends toward a velocity asymptote located between 1.5 and 2. For $x / d_{n}=400$, the tail reaches large values up to $d /\langle d\rangle_{\mathcal{V}}=30$ and is concentrated near this asymptote. For $x / d_{n}=800$, the tail spans only up to $d /\langle d\rangle_{\mathcal{V}}=20$ and seems more dispersed near the asymptote. The reduction of the droplet-size maxima from 30 to 20 can be explained by the ongoing fragmentation process, which globally reduces the size of the water fragments and specifically the largest ones issued from the liquid core breakup. Regarding the velocity dispersion near the asymptote, one has to consider the non-normalized joint PDF in order to 


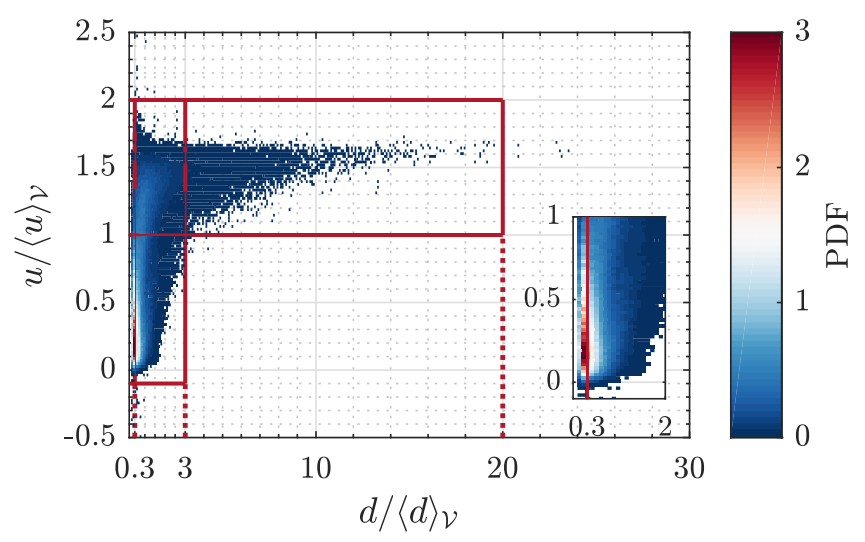

FIG. 9. Size-velocity joint PDF for $x / d_{n}=600$ and droplet subsets discriminated with the characteristics of the size and axial-velocity distributions.

draw a conclusion independent of the evolution of $\langle u\rangle_{\mathcal{V}}$ along $x / d_{n}$, given in Fig. 3. Furthermore, it is possible to observe a "path" of relatively large PDF values, colored in light blue, leaving from the maximum values of the joint PDF, leading to its tail, and following the elbow shape. A close look at the joint PDF for $x / d_{n}=400$ even allows us to observe two paths. Concerning the correlation between $d /\langle d\rangle_{\mathcal{V}}$ and $u /\langle u\rangle_{\mathcal{V}}$, the large-size droplets show little correlation to their velocity and the low-velocity droplets show little correlation to their size, as both sets present a small dispersion along each axis, respectively. However, the in-between range of droplets seems to indicate a strong correlation between the size and the axial velocity.

Different droplet subsets can be derived from the description of the joint PDF. The maximum values of the joint PDF are representative of the most probable pairs $\left(d /\langle d\rangle_{\mathcal{V}}, u /\langle u\rangle_{\mathcal{V}}\right)$ and are concentrated in the region of low velocities and small sizes. Thus, it naturally depicts a first subset of droplets which are likely to behave like passive tracers. A second subset can be drawn by the tail expansion along the $d /\langle d\rangle_{\mathcal{V}}$ axis. The tail along this axis exists only for high velocities and depicts the existence of a group of droplets characterized, at the same time, by a high velocity and a large size, which are likely to behave like ballistic objects. In addition, the joint PDFs show a third region of relatively high PDF values corresponding to the corner of the elbow. This region depicts a subset of droplets characterized by a high velocity and a small or intermediate size. While the first two droplet subsets cross-check the conjecture of Felis et al. [8], the existence of a third droplet subset is brought to light.

Knowing that subgroups with different physical behavior exist in the present droplet population, it could be possible to define them using some characteristics of the PDF of $d /\langle d\rangle_{\mathcal{V}}$ and $u /\langle u\rangle_{\mathcal{V}}$ like the spanning limits, the mode limits, and the dispersion. Consider the joint PDF for $x / d_{n}=600$ as an example. In a straightforward manner, the spanning limits of the PDFs $\mathcal{P}_{d /\langle d\rangle_{\mathcal{V}}}$ and $\mathcal{P}_{u /\langle u\rangle_{\mathcal{V}}}$ give the outward limits of the possible droplet subsets: $0<d /\langle d\rangle_{\mathcal{V}}<20$ and $-0.1<u /\langle u\rangle_{\mathcal{V}}<2$. The modes of $\mathcal{P}_{d /\langle d\rangle_{\mathcal{V}}}$ and $\mathcal{P}_{u /\langle u\rangle_{\mathcal{V}}}$ depict specific sizes and velocities, potentially connected to distinct physical behaviors, and their delimitations can be used to discriminate subsets of the joint PDF. The value $u /\langle u\rangle_{\mathcal{V}}=1$ is chosen to delimit the axial-velocity modes, while the value $d /\langle d\rangle_{\mathcal{V}}=0.3$ is chosen to delimit the size modes. Finally, the delimitation of the size modes and the dispersion zone of $\mathcal{P}_{d /\langle d\rangle_{\mathcal{V}}}$, i.e., its tail, is taken as $3 \sigma_{d} \approx 3$. It is worth noting that the droplets whose size is between 0 and $3 \sigma_{d}$ represent around $96 \%$ of the overall population, a percentage close to the Gaussian dispersion property. The delimitations detailed here are shown on the size-velocity joint PDF for $x / d_{n}=600$ in Fig. 9.

Five subsets appear from those delimitations. Consider first the droplets showing a low axial velocity, i.e., a velocity corresponding to the first velocity mode $u /\langle u\rangle_{\mathcal{V}} \approx 0.2$. Those droplets are 
TABLE IV. Repartition of the droplet volume, number, and Stokes number of the joint PDF subsets at $x / d_{n}=600$.

\begin{tabular}{lccccc}
\hline \hline No. & $u /\langle u\rangle_{\mathcal{V}}$ & $d /\langle d\rangle_{\mathcal{V}}$ & Volume $(\%)$ & Number $(\%)$ & St \\
\hline 1 & {$[0,1]$} & {$[0,0.3]$} & $O\left(10^{-2}\right)$ & 25.2 & 0.83 \\
2 & {$[0,1]$} & {$[0.3,3]$} & 3.69 & 48.8 & 7.92 \\
3 & {$[1,2]$} & {$[0,0.3]$} & $O\left(10^{-3}\right)$ & 1.20 & 0.39 \\
4 & {$[1,2]$} & {$[0.3,3]$} & 10.8 & 22.2 & 7.61 \\
5 & {$[1,2]$} & {$[3,20]$} & 85.5 & 2.55 & 44.0 \\
\hline \hline
\end{tabular}

located in the bottom left corner of the joint PDF, $u /\langle u\rangle_{\mathcal{V}}<1$, and are depicted by two subsets: $\left(d /\langle d\rangle_{\mathcal{V}}, u /\langle u\rangle_{\mathcal{V}}\right)$ in $[0,0.3] \times[-0.1,1]$ and $[0.3,3] \times[-0.1,1]$. Two droplet populations seem to coexist in this range of velocities. Each of them is respectively characterized by a size $d /\langle d\rangle_{\mathcal{V}} \approx 0.2$ with a little dispersion and a size $d /\langle d\rangle_{\mathcal{V}} \approx 0.4$ with a larger dispersion. Thus the droplet set of low velocity and small size pointed out in [8] not only can be defined but is also made of two distinct droplet populations showing different characteristic sizes.

Now consider the droplets showing a high axial velocity, i.e., a velocity corresponding to the second velocity mode $u /\langle u\rangle_{\mathcal{V}} \approx 1$.4. Those droplets are located in the upper part of the joint PDF, $u /\langle u\rangle_{\mathcal{V}}>1$, and are depicted by three subsets: $\left(d /\langle d\rangle_{\mathcal{V}}, u /\langle u\rangle_{\mathcal{V}}\right)$ in $[0,0.3] \times[1,2]$, $[0.3,3] \times[1,2]$, and $[3,20] \times[1,2]$. The top right subset presents a droplet population showing little dispersion in velocity, which increases slightly when the droplet size decreases. That is to say, the droplet size has little impact on the droplet axial velocity. Thus, the fragments of this population tend to decrease in size with an almost constant velocity, which cross-checks the existence of a group of droplets showing a ballisticlike behavior highlighted in [8]. The middle top subset presents relatively high joint PDF values, around 1 . These values highlight the presence of a preferential droplet population. This population is characterized by a high axial velocity $u /\langle u\rangle_{\mathcal{V}} \approx 1.5$ and an intermediate size $d /\langle d\rangle_{\mathcal{V}} \approx 1$. Such velocity and size values respectively correspond to the second velocity mode and the third size mode, clearly visible for $x / d_{n}=400$ in Fig. 2 . In addition, this means as well that the third mode of $\mathcal{P}_{d /\langle d\rangle_{\mathcal{V}}}$, visible for $x / d_{n}=400$, also has importance for higher values of $x / d_{n}$. Finally, the last subset drawn by the chosen delimitations is the one on the top left corner. This subset presents joint PDF values less than 0.5 . Such values are relatively low compared to the values of the nearby subsets, which are 1.5-3 for the bottom left subset and around 1 for the middle top subset. Thus, this subset could be considered as the expression of the tails of the nearby populations instead of depicting a droplet population characterized by a specific size and velocity.

Table IV gives for $x / d_{n}=600$ the repartition of the droplet volume and number in the joint PDF subsets as well as the Stokes number associated with each subset. The low-velocity subsets in Fig. 9, $u /\langle u\rangle_{\mathcal{V}} \leqslant 1$, are denoted by 1 and 2 . The upper subsets are denoted by 3 , 4, and 5 . The ordering reads from left to right. The low-velocity subsets 1 and 2 represent $74 \%$ of the droplets but only $3.7 \%$ of the overall droplet volume. In contrast, for the high-velocity subgroups, subset 5 represents $85 \%$ of the overall droplet volume for only $2.55 \%$ of the droplets. Subset 4 aggregates $22.2 \%$ of the droplet population and $10.8 \%$ of the total volume. Such values are modest, but balanced, and could highlight the role played by this subset to link the populations of large and small droplet size. Finally, subset 3 only represents $1.2 \%$ of the population and a relative volume of $O\left(10^{-3}\right)$. Such values are negligible compared to the other subsets and they support the interpretation of a subset being the "tail" of its neighbors.

The Stokes number St of each subset is calculated with the formulation from [10,41], using the Schiller-Naumann relation for the drag coefficient and the averaging operator $\langle\cdot\rangle_{\mathcal{V}}$, which is written as

$$
\mathrm{St}=\frac{\tau_{d}}{\tau_{t}}, \quad \tau_{d}=\frac{\rho_{l} d^{2}}{18 \mu_{g}\left(1+0.15 \operatorname{Re}_{d}^{0.687}\right)}, \quad \tau_{t}=\frac{y_{0.5 u}}{\sqrt{\bar{R}_{11, g}}}, \quad \operatorname{Re}_{d}=\frac{\left|u-\bar{u}_{g}\right| d}{v_{G}},
$$


TABLE V. Mean velocity values on the jet axis from the DTV measurements.

\begin{tabular}{lc}
\hline \hline$x / d_{n}$ & $u_{\text {axis }}(\mathrm{m} / \mathrm{s})$ \\
\hline 400 & 33.2 \\
500 & 31.8 \\
600 & 30.4 \\
700 & 29.0 \\
800 & 28.0 \\
\hline \hline
\end{tabular}

where $\operatorname{Re}_{d}$ is the Reynolds number seen by the droplet calculated using the gas mean velocity $\bar{u}_{g}$, $\tau_{d}$ is the droplet aerodynamic time constant, and $\tau_{t}$ is a turbulent timescale estimated from the axial standard deviation for the velocity fluctuations in the gas phase $\sqrt{\bar{R}_{11, g}}$ and the radial position $y_{0.5 u}$ at which the fluid velocity is half the fluid velocity on the jet axis. The five droplet subsets are distributed over two decades of St and show three different trends. The Stokes number of subset 5, containing the droplets of high velocity and large size, reaches 44 and is representative of a ballistic behavior. The Stokes-number values for subsets 2 and 4 are between 7 and 8 . As these values are larger than but close to unity, the droplets belonging to those subsets are in a ballistic regime but might be sensitive to large velocity fluctuations due to turbulence. Subsets 1 and 3 show a Stokes number less than unity, which makes those droplets the most sensitive to turbulence fluctuations. However, the droplets of this range might be unresponsive to the smallest scales of turbulence, as both Stokes numbers are relatively close to 1 . It is important to note here that the velocity has little influence on the Stokes number. For example, subsets 2 and 4 present similar Stokes numbers whereas they are characterized by different velocities. The observation holds for subsets 1 and 3 as well, even if a slight departure due to the velocity difference is noticeable.

In addition to validating the conjecture drawn by Felis et al. [8] regarding the existence of two different droplet subgroups, this section precisely depicts and characterizes such groups among the overall droplet population. Specifically, a precise criterion is given for the low-velocity droplets which leads to the discrimination of two populations with different characteristic sizes. Furthermore, a subset of droplets showing high axial velocity and intermediate size is depicted and characterized. Complementarily, the weight of each subset in terms of droplet number and volume is given along with their Stokes number. Knowing the different subsets in the present droplet population now opens the way to understanding the underlying mechanisms leading to their existence.

\section{B. Quadratic formula for joint PDF modeling}

Section II C presented the work of Lee and An [20] and the quadratic formula (21) they derived to depict the relationship between the droplet velocity and the Sauter mean diameter $d_{3,2}$ for $x / d_{n} \leqslant$ 100. In their study, the authors concluded that the good performance of the relationship enabled them to reproduce experimental and numerical data. In particular, this relationship is said to perform a good fitting of the centerline of a droplet-size-velocity joint PDF from Rimbert and Castanet [42], even if no mathematical definition of a joint PDF centerline is given. Finally, the authors claimed that the droplet-size PDF can be reconstructed from the diameters computed due to the quadratic formula. This section investigates the ability of this quadratic formula to fit the centerline of the present size-velocity joint PDF and the possibility to reconstruct the droplet-size distribution for $x / d_{n} \geqslant 400$. This investigation will consider the diameter $d$ instead of $d_{3,2}$, as it is the available quantity in the present study for characterizing the droplet diameter. In addition, two reference velocities will be compared: the injection velocity $u_{i n j}$ and the mean liquid velocity on the jet axis at each $x / d_{n}$, denoted by $u_{\text {axis }}$. The values of the latter for each axial position are given in Table V. 

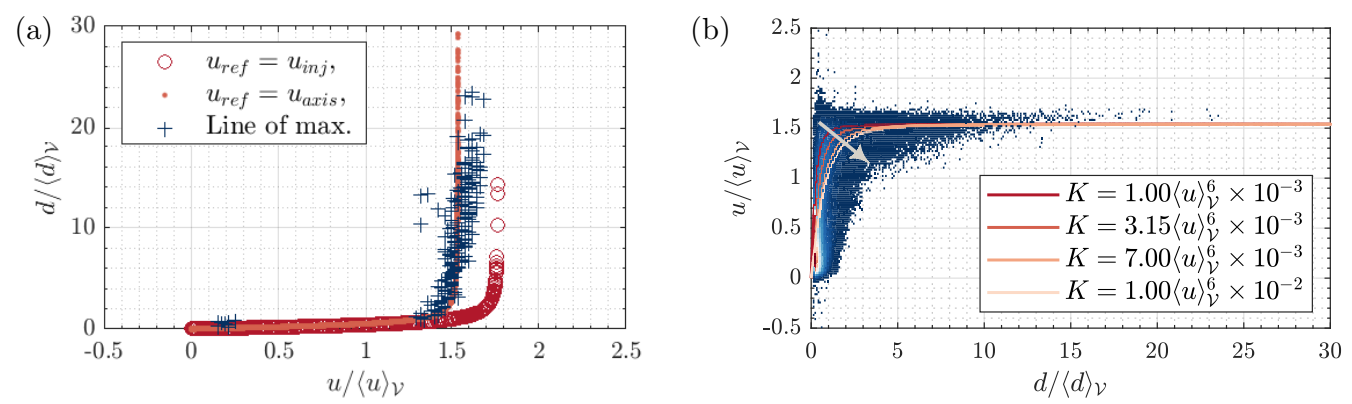

FIG. 10. (a) Comparison of two reference velocities for fitting the centerline of the size-velocity joint PDF at $x / d_{n}=600$ by Eq. (27) where the fit parameter $K$ is set to $3.15\langle u\rangle_{\mathcal{V}}^{6} \times 10^{-3}$. (b) Comparison of different values of $K$ for fitting the centerline of the size-velocity joint PDF for $x / d_{n}=600$ by Eq. (27). The arrow indicates increasing values of the fitting parameter $K$.

The reference velocity is denoted by $u_{r e f}$ and Eq. (21) is rewritten as

$$
d=\frac{3 \sigma_{l-g}+\sqrt{9 \sigma_{l-g}^{2}+K \rho_{l} \mu_{l} \bar{u}^{2} \frac{u_{r e f}^{2}-u^{2}}{2}}}{\rho_{l} \frac{u_{r e f}^{2}-u^{2}}{2}} .
$$

Let us tackle first what the centerline of a joint PDF is. Considering the fitting of the joint PDF from Lee and An [20], the centerline of a joint PDF seems to be a line of PDF values such that it describes the main trend of the two-dimensional map. Here the centerline would correspond to the path of relatively large PDF values, visible in Fig. 8 and mentioned in the preceding section. The centerline would then correspond to local maximum values along each direction and could be defined by the zero values of the joint PDF gradient. As this path of relatively large PDF values spans large values of $d$, the gradient should be computed along $d$ to capture all of the centerline. Thus, it could be possible to define the joint PDF centerline as the zero isoline of the joint PDF gradient computed along $d$.

Technically, there is an ambiguity when using the latter definition as the borders of the joint PDF also present gradient values close to zero. As a first approach, the centerline of the joint PDF is defined as the local maximum values of the PDF and is captured by searching for the local maximum in each bin along the direction $d$. The centerline produced by this approach is given in Fig. 10 and is referred to as the line of maxima. It is possible to observe a jump from $u /\langle u\rangle_{\mathcal{V}}=0.2$ to $u /\langle u\rangle_{\mathcal{V}}=1.4$ which is due to the existence of a local maximum in the region of low velocity and small size, corresponding to the bottom right subset in Fig. 9. Even if this estimation of the joint PDF centerline presents a discontinuity, it depicts well the overall trend of the joint PDF. Using this estimation, it is possible to compare the two reference velocities $u_{i n j}$ and $u_{\text {axis }}$ for fitting the joint PDF centerline. A fitting procedure is carried out for the fit parameter $K$ and gives a value of $3.15\langle u\rangle_{\mathcal{V}}^{6} \times 10^{-3}$. It appears in Fig. 10 that using the injection velocity as a reference overestimates the centerline. In contrast, using the mean liquid velocity on the jet axis leads to a more satisfactory result. The latter velocity $u_{\text {axis }}$ is then used in the following as the reference velocity. In addition, it also appears that the quadratic formula proposed by Lee and An [20] performs nicely to capture the trend of the present joint PDF at large $x / d_{n}$, while it was initially derived for $d_{3,2}$ at small $x / d_{n}$ distances.

Figure 10 presents the droplet-size velocity at $x / d_{n}=600$ and Eq. (27) for different values of $K$. It can be seen that Eq. (27) qualitatively depicts the trend of the joint PDF for values of $K$ between $1.00 \times 10^{-3}\langle u\rangle_{\mathcal{V}}$ and $1.00 \times 10^{-2}\langle u\rangle_{\mathcal{V}}$. This questions the value of $K$ chosen to model the centerline in Fig. 10, especially under the limitation of discontinuity of the previous estimation. 

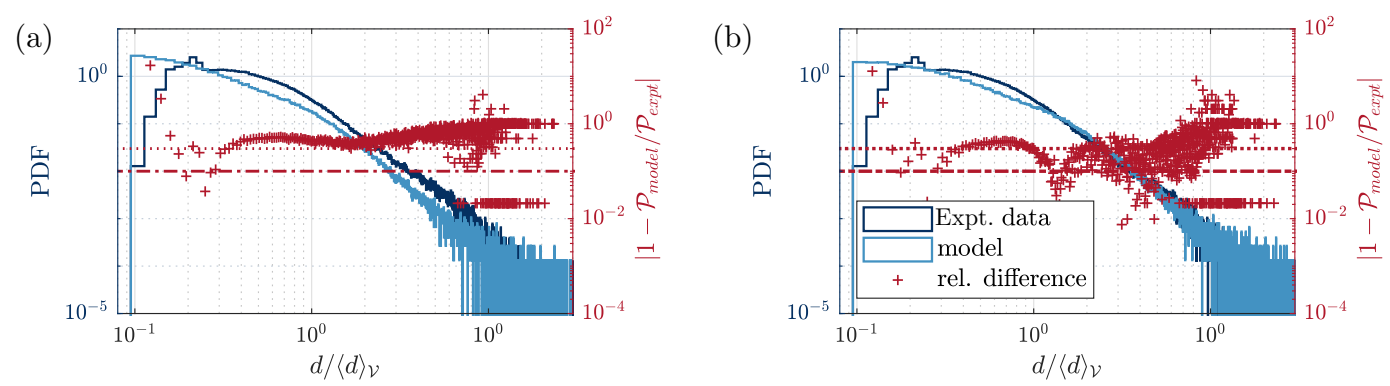

FIG. 11. Comparison of the experimental size PDF and the PDF reconstructed from the size given by Eq. (27) with (a) $K=3.15\langle u\rangle_{\mathcal{V}}^{6} \times 10^{-3}$ and (b) $K=7.00\langle u\rangle_{\mathcal{V}}^{6} \times 10^{-3}$. The pluses indicate the relative difference between the two PDFs. The dotted and dashed lines represent relative differences of 0.3 and 0.1 , respectively.

In order to figure out a physically meaningful value of $K$, one could have a look at the reconstruction of the droplet-size PDF from the sizes given by Eq. (27). Figure 11 compares the experimental droplet-size PDF with the reconstructed PDF for two values of $K: 3.15\langle u\rangle_{\mathcal{V}}^{6} \times 10^{-3}$ and $7.00\langle u\rangle_{\mathcal{V}}^{6} \times 10^{-3}$. In addition to comparing the experimental and model PDF, Fig. 11 gives the relative difference between the two, defined as $\left|1-\mathcal{P}_{\text {model }} / \mathcal{P}_{\text {expt }}\right|$ and represented by the red pluses. An estimation is commonly considered as acceptable when the relative difference is less than $30 \%$ and considered as relatively good when it is less than $10 \%$. Those two thresholds are indicated in Fig. 11 as well. On the one hand, the droplet-size PDF reconstructed with $K=3.15\langle u\rangle_{\mathcal{V}}^{6} \times 10^{-3}$ reproduces a trend similar to the experimental PDF but shows an almost constant offset with a relative difference systematically larger than 0.3 . On the other hand, the PDF reconstructed with $K=7.00\langle u\rangle_{\mathcal{V}}^{6} \times 10^{-3}$ reproduces as well a trend similar to the experimental PDF and presents a smaller offset than previously. The relative difference is less than 0.3 up to $d /\langle d\rangle_{\mathcal{V}} \approx 3$, where the distribution tail begins. However, in both cases, the relative difference between the two PDF tails presents large values, up to 10, and the model PDF is not able to capture the two modes for $d /\langle d\rangle_{\mathcal{V}} \approx$ 0.2 and 0.4 . The box-and-whisker plot of the relative difference with $K=7.00\langle u\rangle_{\mathcal{V}}^{6} \times 10^{-3}$ reveals that almost $50 \%$ of the relative difference values are under 0.3 . It is worth noting that excluding the PDF tail would improve this result as most of the large values of relative difference are recorded in the tail region, whereas it represents only $4 \%$ of the total droplet number. Thus, the latter value of $K$ enables us to estimate a joint PDF centerline which produces a droplet-size PDF close to the experimental one.

Even if Eq. (27) were derived in the context of turbulent combustion, i.e., for small $x / d_{n}$ distances, and using the Sauter mean diameter $d_{3,2}$, good agreement is found by depicting the trend of the droplet-size-velocity joint PDF under the condition that the mean velocity on the jet axis $u_{\text {axis }}$ is used as a reference. In addition, this formulation is able to produce a droplet-size PDF with a trend similar to the experimental one, if the value of the fitting parameter $K$ is chosen in order to minimize the difference between the model and the experimental PDF. However, the model PDF fails to capture the first two modes in size.

\section{CONCLUSION}

In this work, it has been shown that, for agricultural-like jets lying in the second-wind-induced regime [1], both the droplet-size and axial-velocity distributions present distinct modes. On the one hand, the size distribution presents three modes for $d /\langle d\rangle_{\mathcal{V}} \in\{0.2,0.4,1\}$, the second one corresponding to the Taylor scale, and a large dispersion towards $d /\langle d\rangle_{\mathcal{V}}=30$ with a decay scaling as $d /\langle d\rangle_{\mathcal{V}}^{-2.7}$. On the other hand, the axial-velocity distribution presents two modes for $u /\langle u\rangle_{\mathcal{V}} \in\{0.2,1.4\}$ and a little dispersion. The dispersion of the latter is limited by two boundary conditions: the global advection of the water fragments and the mean axial velocity on the jet axis. 
Regarding the distribution of $v /\langle u\rangle_{\mathcal{V}}$, only one mode appears to exist and the distribution seems to behave in a more classical manner, similar to what can be found in the turbulence literature. Close similarity is observed for each distribution over the axial positions. Two theories have been tested to model the droplet-size distribution. The first one comes from the work of Villermaux et al. [22] based on a fine analysis of the ligament dynamics in low-turbulence fragmentation flows while the second one comes from Novikov and Dommermuth [15], who used intermittency in turbulence. In the limits of this study, it appears that, for $x / d_{n} \geqslant 400$, the distribution proposed by Novikov and Dommermuth [15] describes better the experimental size distribution and is able to capture the two modes at small $d /\langle d\rangle_{\mathcal{V}}$. Regarding the modeling of the axial-velocity distribution, only the mode of low velocity $u /\langle u\rangle_{\mathcal{V}} \approx 0.2$ exists in the dispersion zone of the jet. Among the three distributions derived for combustion applications, this mode is better depicted by the log-normal distribution. Taking benefit from this insight, a model PDF was proposed to depict the overall distribution of $u /\langle u\rangle_{\mathcal{V}}$ at a given $x / d_{n}$. It does not capture the tails of the experimental distribution but describes nicely the two velocity modes, i.e., more than $50 \%$ of the overall droplet population.

Multimodal distributions suggest that different characteristic groups could exist in the droplet population. The analysis of the droplet-size-velocity joint PDF highlights the existence of three different behaviors. Two of them cross-check the conjecture of Felis et al. [8], who highlighted the existence of droplets behaving like passive tracers, with a small size and high-velocity fluctuations, or like ballistic objects, with a large size, high mean velocity, and almost zero fluctuations. In addition, this analysis reveals the existence of a third droplet group, characterized by a high axial velocity and an intermediary range of size. Using the characteristics of the size and axial-velocity PDF such as the spanning limits, the mode limits, and the distribution dispersion, it is possible to characterize precisely five subsets of droplets. Four seem to depict droplet groups with specific characteristic size and velocity while the last one seems to be the expression of the tails of the nearby populations. The Stokes numbers of those subsets follow three trends and span from 0.39 to 44 , which corroborates the ballistic and passive tracer behaviors. However, this quantity presents a little dependence on the droplet velocity and is not able to discriminate droplet subsets with similar characteristic velocities. In the combustion framework, Lee and An [20] derived a quadratic formula to model the centerline of the joint PDF. After proposing a mathematical definition of such a centerline, the formula was tested on the present experimental data. It qualitatively described well the trend of the joint PDF. The size PDF reconstructed from this formula offers an acceptable description of the experimental data but fails to capture the two small size modes. It appears that results from combustion studies, developed in the region close to the nozzle, are valid as well in the dispersion region of agricultural-like configurations, i.e., far away from the nozzle.

Knowing the most probable droplet groups in the present flow opens the way to better understand the mechanisms at work. Several questions remain open. Which mechanism is responsible of the small-droplet-size mode? Which mechanism do the largest droplets undergo? Which mechanism produces such a power-law decay in the droplet-size distribution? Further works could focus on analyzing the turbulent energy spectra or on characterizing the droplet geometry in each subset of the size-velocity joint PDF to target such mechanisms. Complementary works could also be achieved to numerically reproduce similar configurations and investigate the ligament dynamics close to the nozzle.

\section{ACKNOWLEDGMENTS}

The authors thank F. Felis-Carasco and S. Tomas for making their data available. R.V. thanks E. Villermaux for fruitful discussion and comments.

[1] C. Dumouchel, On the experimental investigation on primary atomization of liquid streams, Exp. Fluids 45, 371 (2008). 
[2] G. M. Faeth, Structure and breakup properties of spray, Int. J. Multiphase Flow 21, 99 (1995).

[3] P. K. Wu and G. M. Faeth, Aerodynamic effects on primary breakup of turbulent liquids, Atomization Spray. 3, 265 (1993).

[4] P. K. Wu and G. M. Faeth, Onset and end of drop formation along the surface of turbulent liquid jets in still gases, Phys. Fluids 7, 2915 (1995).

[5] P. K. Wu, R. Miranda, and G. M. Faeth, Effects of initial flow conditions on primary breakup of nonturbulent and turbulent round liquid jets, Atomization Spray. 5, 175 (1994).

[6] P. K. Wu, L. K. Tseng, and G. M. Faeth, Primary breakup in gas/liquid mixing layers for turbulent liquids, Atomization Spray. 2, 295 (1992).

[7] C. Stevenin, S. Tomas, A. Vallet, M. Amielh, and F. Anselmet, Flow characteristics of a large-size pressure-atomized spray using DTV, Int. J. Multiphase Flow 84, 264 (2016).

[8] F. Felis, S. Tomas, A. Vallet, M. Amielh, and F. Anselmet, Experimental analysis of the flow characteristics of a pressure-atomized spray, Int. J. Heat Fluid Flow 85, 108624 (2020).

[9] E. Ruffin, R. Schiestel, F. Anselmet, M. Amielh, and L. Fulachier, Investigation of characteristic scales in variable density turbulent jets using a second-order model, Phys. Fluids 6, 2785 (1994).

[10] F. Felis-Carrasco, Atomization and dispersion of a liquid jet: Numerical and experimental approaches, Ph.D. thesis, Ecole Centrale Marseille, 2017.

[11] E. Villermaux, Fragmentation, Annu. Rev. Fluid Mech. 39, 419 (2007).

[12] P. G. M. Marmottant and E. Villermaux, On spray formation, J. Fluid Mech. 498, 73 (2004).

[13] J. Eggers and E. Villermaux, Physics of liquid jets, Rep. Prog. Phys. 71, 036601 (2008).

[14] E. Villermaux and B. Bossa, Single-drop fragmentation determines size distribution of raindrops, Nat. Phys. 5, 697 (2009).

[15] E. A. Novikov and D. G. Dommermuth, Distribution of droplets in a turbulent spray, Phys. Rev. E 56, 5479 (1997).

[16] E. A. Novikov, Infinitely divisible distributions in turbulence, Phys. Rev. E 50, R3303 (1994).

[17] E. A. Novikov, Intermittency and scale similarity in the structure of a turbulent flow, J. Appl. Math. Mech. 35, 231 (1971).

[18] N. Rimbert and O. Sero-Guillaume, Log-stable laws as asymptotic solutions to a fragmentation equation: Application to the distribution of droplets in a high Weber-number spray, Phys. Rev. E 69, 056316 (2004).

[19] N. Rimbert and G. Castanet, Crossover between Rayleigh-Taylor instability and turbulent cascading atomization mechanism in the bag-breakup regime, Phys. Rev. E 84, 016318 (2011).

[20] T.-W. Lee and K. An, Quadratic formula for determining the drop size in pressure-atomized sprays with and without swirl, Phys. Fluids 28, 063302 (2016).

[21] O. Simonin, in Combustion and Turbulence in Two-Phase Flows: Continuum Modelling of Dispersed Two-Phase Flows, edited by M. Manna, VKI Lecture Series (von Karman, Institute for Fluid Dynamics, Sint-Genesius-Rode, 1996).

[22] E. Villermaux, P. G. M. Marmottant, and J. Duplat, Ligament-Mediated Spray Formation, Phys. Rev. Lett. 92, 074501 (2004).

[23] M. Von Smoluchowski, Versuch einer mathematischen theorie der koagulationskinetik kolloider lösungen, Z. Phys. Chem. 92, 129 (1917).

[24] S. K. Friedlander, Smoke, Dust, and Haze: Fundamentals of Aerosol Dynamics, 2nd ed. (Oxford University Press, New York, 2000).

[25] E. Villermaux and B. Bossa, Drop fragmentation on impact, J. Fluid Mech. 668, 412 (2011).

[26] S. Kooij, R. Sijs, M. M. Denn, E. Villermaux, and D. Bonn, What Determines the Drop Size in Sprays? Phys. Rev. X 8, 031019 (2018).

[27] A. N. Kolmogorov, The local structure of turbulence in an incompressible fluid for very large Reynolds numbers, Dokl. Akad. Nauk SSSR 30, 299 (1941).

[28] A. N. Kolmogorov, Energy dissipation in locally isotropic turbulence, Dokl. Akad. Nauk SSSR 32, 19 (1941).

[29] L. Landau and E. M. Lifshitz, Fluid Mechanics, A Course of Theoretical Physics Vol. 6 (Pergamon, Oxford, 1959). 
[30] A. N. Kolmogorov, Colloques Internationaux du Centre National de la Recherche Scientifique (CNRS, Paris, 1961), Vol. 108, pp. 447-458.

[31] A. N. Kolmogorov, A refinement of previous hypotheses concerning the local structure of turbulence in a viscous incompressible fluid at high Reynolds number, J. Fluid Mech. 13, 82 (1962).

[32] A. M. Obukhov, Some specific features of atmospheric turbulence, J. Fluid Mech. 13, 77 (1962).

[33] U. Frisch, Turbulence: The Legacy of A.N. Kolmogorov (Cambridge University Press, Cambridge, 1995).

[34] F. Anselmet, R. A. Antonia, and L. Danaila, Turbulent flows and intermittency in laboratory experiments, Planet. Space Sci. 49, 1177 (2001).

[35] W. Feller, An Introduction to Probability Theory and its Applications, 3rd ed. (Wiley, New York, 1971), Vol. 2.

[36] H. Tennekes and J. L. Lumley, A First Course in Turbulence (MIT Press, Cambridge, 1972).

[37] H. C. Simmons, The correlation of drop-size distributions in fuel nozzle sprays. Part II. The dropsize/number distribution, J. Eng. Power 99, 315 (1977).

[38] J. W. Hoyt and J. Taylor, Waves on water jet, J. Fluid Mech. 83, 119 (1977).

[39] F. Moisy, EzyFit 2.44 (The MathWorks, Inc., Natick, 2020).

[40] S. S. Yoon, Droplet distributions at the liquid core of a turbulent spray, Phys. Fluids 17, 035103 (2005).

[41] V. Ferrand, R. Bazile, J. Borée, and G. Charnay, Gas-droplet turbulent velocity correlations and two-phase interaction in an axisymmetric jet laden with partly responsive droplets, Int. J. Multiphase Flow 29, 195 (2003).

[42] N. Rimbert and G. Castanet, Liquid atomization out of a full cone pressure swirl nozzle, arXiv:1008.2474. 